\title{
Nonlinear filtering with correlated Lévy noise characterized by copulas
}

\author{
B. P. W. Fernando and E. Hausenblas \\ Montanuniversitaet Leoben
}

\begin{abstract}
The objective in stochastic filtering is to reconstruct the information about an unobserved (random) process, called the signal process, given the current available observations of a certain noisy transformation of that process.

Usually $X$ and $Y$ are modeled by stochastic differential equations driven by a Brownian motion or a jump (or Lévy) process. We are interested in the situation where both the state process $X$ and the observation process $Y$ are perturbed by coupled Lévy processes. More precisely, $L=\left(L_{1}, L_{2}\right)$ is a 2dimensional Lévy process in which the structure of dependence is described by a Lévy copula. We derive the associated Zakai equation for the density process and establish sufficient conditions depending on the copula and $L$ for the solvability of the corresponding solution to the Zakai equation. In particular, we give conditions of existence and uniqueness of the density process, if one is interested to estimate quantities like $\mathbb{P}(X(t)>a)$, where $a$ is a threshold.
\end{abstract}

\section{Introduction}

The objective in stochastic filtering is to reconstruct information about an unobserved (random) process, called the signal process, given the current available observations of a certain noisy transformation of that process. Here, the underlying problem is, that the unobserved problem may be corrupted by noise, and in addition, the observations made are usually again corrupted by some noise or random errors. The main objective of stochastic filtering is to estimate an evolving dynamical system usually called signal. That is, to extract the most precise information about the underlying system and to filter out the "noise" in the observations. These kind of problem appears in physics, engineering, and finance among others.

This measurement noise is modeled very often by a stochastic process of Gaussian or Poisson type. In particular, the signal and the observation process can be modeled either by a discontinuous or continuous random process. When both the signal $X$ and the observation $Y$ have discontinuous paths, one can distinguish three main frameworks. The first one is the case in which $Y$ is driven by a counting process or a marked point process. We can refer to Brémaud (1981), Ceci (2006), Frey and Runggaldier (2001), Kliemann, Koch and Marchetti (1990), Lipster and

Key words and phrases. Nonlinear filtering, Lévy processes, Lévy copula.

Received June 2015; accepted November 2016. 
Shiryaev $(1989,2001)$ among others for the results and advances made in this situation. The second framework is the case in which $Y$ is driven by a mixed type process, that it, $Y$ can be viewed as a sum of marked point process and a diffusion process. This case is the subject of recent papers of Ceci and Colaneri (2012), Frey and Runggaldier (2010), Frey and Schimdt (2012), Frey, Schimdt and Xu (2013). Finally, one can model the signal $X$ and the observation $Y$ by a jump-diffusion processes, which is done for example, in Ceci and Colaneri (2012). In that works, they also allow processes $X$ and $Y$ to be correlated and have common jump times.

In the present paper, we consider the filtering problem similar to the model in Ceci and Colaneri (2012) but address the difficult situation where the signal and observation process are driven by two Lévy processes which are correlated. To be more precise, in our model the state $X$ and the observable $Y$ solve a stochastic equation driven by general Lévy processes. The Brownian part in $X$ may be degenerate. In addition, both processes are corrupted by a pair of two purely discontinuous Lévy processes, where the dependence structure is given by a Lévy copula. Here $X$ is corrupted by the first process and the observation process is corrupted by the second process. By using the change of measure method we derive the associated Zakai equation. Using copula, we were able to calculate the diffusion coefficient in front of the random driving process in the Zakai equation explicitly. We treat the case of finite and infinite Lévy measure separately in Theorem 2.2 and Theorem 2.3. As mentioned in the abstract, we were mainly interested in the case where one would like to estimate entities like $\mathbb{P}(X(t)>a \mid Y(s), 0 \leq s \leq t)=\mathbb{E}\left[1_{(a, \infty)}(X(t)) \mid Y(s), 0 \leq s \leq t\right], a \in \mathbb{R}$. Here the main difficulty is that the function $\mathbb{R} \ni x \mapsto 1_{(a, \infty)}(x)$ is not twice differentiable and one has to use the smoothing property of the infinitesimal generater of the driving Lévy process of $X$ (see Fernando Pani, Hausenblas and Razafimandimby (2017)). Because of this, we also use the change of measure transformation and consider the Zakai equation. In this paper, we were able to specify in Theorem 3.1 the exact conditions under which the density process exists and is uniquely defined. In addition, we investigated the regularity of the process.

The organization of the paper is as follows. In Section 2, we introduce the problem and derive the Zakai equation for finite and infinite Lévy measures. In Section 3, we consider the case where one is interested to estimate an entity like $\mathbb{P}(X(t)>a), a \in \mathbb{R}$. Here, the main result is Theorem 3.1. Corollary 3.1 is an example which illustrates the applicability of Theorem 3.1. In the appendix, we summarize results that are necessary for the proofs of our main results. In particular, in Appendix A we introduce the Zakai equation as an evolution equation taking values in Sobolev spaces. In Appendix B, we introduce Lévy copulas and give known results necessarily for the proofs of our main results.

Notation 1.1. We denote by $\mathbb{R}_{+}$the positive real half line, i.e. $\mathbb{R}_{+}=(0, \infty)$, and by $\mathbb{R}_{+}^{0}$ the positive real have line including zero, that is, $\mathbb{R}_{+}^{0}=[0, \infty)$. For a mea- 
surable space $(E, \mathcal{E})$, we denote by $B_{b}(E)$ the Banach space of all bounded, realvalued, $\mathcal{E}$-measurable functions equipped with the supremum norm. For a metric space $(E, \mathcal{E})$, we denote by $C_{b}(E)$ the Banach space of all bounded, real-valued and continuous functions equipped with the supremum norm. Let us denote by $\mathcal{S}$ the Schwarz space of all rapidly decreasing functions and $\mathcal{S}^{\prime}$ its dual. For $s \in \mathbb{R}$ and $p \geq 1$ we denote by $H_{p}^{s}\left(\mathbb{R}^{d}\right.$ ) the Bessel Potential Spaces (or Sobolev spaces of fractional order), that is,

$$
H_{p}^{s}\left(\mathbb{R}^{d}\right):=\left\{f \in \mathcal{S}^{\prime}:|f|_{H_{p}^{s}}:=\left|\mathcal{F}^{-1}\left(1+|\xi|^{2}\right)^{\frac{s}{2}} \mathcal{F} f\right|_{L^{p}}<\infty\right\} .
$$

Here, $\mathcal{F}$ denotes the Fourier transform given by

$$
\mathcal{F} f(\xi)=\hat{f}(\xi)=(2 \pi)^{-d} \int_{\mathbb{R}^{d}} e^{i \xi^{T} x} f(x) d x, \quad f \in L^{2}\left(\mathbb{R}^{d}\right) .
$$

The space $C_{b}^{(n)}(\mathbb{R})=\{f: \mathbb{R} \rightarrow \mathbb{R}: f$ is $n$ times continuously differentiable and bounded\}.

\section{Problem setting and the Zakai equation}

As mentioned in the introduction, we consider the filtering problem with Lévy noise. In particular, the state and observation processes are both perturbed by a Lévy noise. Since in practice the noises in the state process and the observation process are usually depending on each other, so we allow our model to have certain dependence structure.

In the case of Gaussian variables, the dependence structure is described via a correlation matrix. However for the non-Gaussian random variables, the use of correlation coefficients is often misleading. Hence, we must choose the right tool to describe the dependence structure for non-Gaussian noise. Here, copulas are nowadays widely used in finance to express dependence of non-Gaussian random variables. In Appendix B, we give a short summary on copula and some facts that we need for the proof of our main results. For a more detailed introduction, we refer to the books by Cherubini, Luciano and Vecchiato (2004), Nelsen (2006), Malevergne and Sornette (2006).

Let $\left(X_{1}, X_{2}, \ldots, X_{n}\right)$ be a random vector with marginal distribution functions $F_{i}$, that is, $F_{i}(x)=\mathbb{P}\left(X_{i} \leq x\right)$. By assuming $F_{1}, \ldots, F_{n}$ are continuous, one can show that up to a transformation the random vector

$$
\left(F_{1}\left(X_{1}\right), F_{2}\left(X_{2}\right), \ldots, F_{n}\left(X_{n}\right)\right)
$$

has uniformly distributed margins. The cumulative distribution function $\left(U_{1}, U_{2}\right.$, $\left.\ldots, U_{n}\right)$ associated to $\left(X_{1}, \ldots, X_{n}\right)$ is defined by $U_{i}=F_{i}\left(X_{i}\right), i=1, \ldots, n$. For any random vector $\left(X_{1}, X_{2}, \ldots, X_{n}\right)$ with distribution $F: \mathbb{R}^{n} \rightarrow[0,1]$ and continuous marginal distribution functions $F_{i}$, the function

$$
C:[0,1]^{n} \rightarrow[0,1]
$$


such that

$$
C\left(u_{1}, \ldots, u_{n}\right)=F\left(F_{1}^{-1}\left(u_{1}\right), \ldots, F_{n}^{-1}\left(u_{n}\right)\right), \quad u_{i} \in[0,1], i=1, \ldots, n,
$$

is called the copula. The existence of a copula $C$ associated to given marginal distribution $F_{1}, \ldots, F_{n}$ is ensured by following theorem.

Theorem 2.1 (Sklar's theorem). Given an $n$-dimensional distribution function $F$ with continuous (cumulative) marginal distributions $F_{1}, \ldots, F_{n}$, there exists a unique $n$-copula $C:[0,1]^{n} \rightarrow[0,1]$ such that

$$
F\left(x_{1}, \ldots, x_{n}\right)=C\left(F_{1}\left(x_{1}\right), \ldots, F_{n}\left(x_{n}\right)\right) \quad \forall\left(x_{1}, \ldots, x_{n}\right) \in \mathbb{R}^{n} .
$$

There are several different types of copulas. The ones very frequently seen in the literature are the independent copula defined by

$$
C\left(u_{1}, u_{2}, \ldots, u_{n}\right)=u_{1} u_{2} \cdots u_{n},
$$

the Clayton copula defined for $\theta \in[-1, \infty) \backslash\{0\}$ by

$$
C\left(u_{1}, u_{2}, \ldots, u_{n}\right)=\max \left(\left[u_{1}^{-\theta}+u_{2}^{-\theta}+\cdots+u_{n}^{-\theta}-(n-1)\right]^{-\frac{1}{\theta}}, 0\right),
$$

and the Gumpel copula defined for $\theta \in[1, \infty)$ by

$$
\begin{aligned}
& C\left(u_{1}, u_{2}, \ldots, u_{n}\right) \\
& \quad=\exp \left(-\left[\left(-\ln u_{1}\right)^{\theta}+\left(-\ln u_{2}\right)^{\theta}+\cdots+\left(-\ln u_{n}\right)^{\theta}\right]^{\frac{1}{\theta}}\right) .
\end{aligned}
$$

In a similar way, we can define the Lévy copulas which is a general concept to capture jump dependence in multivariate Lévy processes. The Lévy copula is described in terms of the Lévy measure. For more detailed introduction to Lévy copula, we refer to the works of Cont and Tankov $(2004,2006)$ and Kallsen and Tankov (2006). In addition, we summarize some basic facts in Appendix B. Since the Lévy measure is usually $\sigma$-finite, the definition of a copula has to be extended to a function acting on $[-\infty, \infty]$.

For this purpose, let $v$ be a Lévy measure on $\mathbb{R}^{n}$ with marginal intensities $v_{1}, v_{2}, \ldots, v_{n}$. Let $\mathcal{I}: \mathbb{R} \backslash\{0\} \rightarrow \mathcal{B}(\mathbb{R})$ be given by

$$
\mathcal{I}(x)= \begin{cases}(x, \infty) & x>0, \\ (-\infty, x), & x<0 .\end{cases}
$$

Let $U_{i}$ be the tail integral defined by

$$
U_{i}(z)= \begin{cases}\operatorname{sgn}(z) v_{i}(\mathcal{I}(z)) & \text { for } z \in \mathbb{R} \backslash\{0\} \\ 0 & \text { for } z=\infty \text { or } z=-\infty \\ \infty & \text { for } z=0, i=1,2, \ldots, n\end{cases}
$$


and

$$
\begin{aligned}
& U\left(z_{1}, z_{2}, \ldots, z_{n}\right) \\
& \quad= \begin{cases}\left(\prod_{i=1}^{n} \operatorname{sgn}\left(z_{i}\right)\right) v\left(\prod_{i=1}^{n} \mathcal{I}\left(z_{i}\right)\right) & \text { for } z_{1}, z_{2}, \ldots, z_{n} \in \mathbb{R} \backslash\{0\}, \\
0 & \text { for }\left|z_{i}\right|=\infty, i=1, \ldots, n, \\
v\left(\mathbb{R}^{n}\right) & \text { for } z_{i}=0, i=1, \ldots, n .\end{cases}
\end{aligned}
$$

Now, for an $n$-dimensional Lévy process $L$, one can associate a Lévy copula $H$ : $[-\infty, \infty]^{n} \rightarrow[-\infty, \infty]$ as

$$
U\left(z_{1}, \ldots, z_{n}\right)=H\left(U_{1}\left(z_{1}\right), \ldots, U_{n}\left(z_{n}\right)\right), \quad z_{1}, \ldots, z_{n} \in \mathbb{R} .
$$

In fact, thanks again to Sklar-type theorem (see Kallsen and Tankov (2006), Theorem 3.6) for each $n$-dimensional Lévy process with intensity $v$ and marginal intensities $v_{i}, i=1, \ldots, n$, there exists a Lévy copula $H$ such that

$$
U\left(z_{1}, \ldots, z_{n}\right)=H\left(U_{1}\left(z_{1}\right), \ldots, U_{n}\left(z_{n}\right)\right), \quad z_{1}, \ldots, z_{n} \in \mathbb{R} .
$$

Now, let us proceed with the setting of our main problem. Let $H$ be a Lévy copula and $L=\left\{L(t)=\left(L_{1}(t), L_{2}(t)\right) \in \mathbb{R}^{2}: t \geq 0\right\}$ be a two dimensional pure jump Lévy process with its marginal intensities $v_{1}$ and $v_{2}$. Let $L_{0}$ be a compensated pure jump Lévy process and $W_{2}=\left\{W_{2}(t): t \geq 0\right\}$ be a Brownian motion. We assume that all these objects are defined on a probability space $\mathfrak{A}=\left(\Omega, \mathcal{F},\left(\mathcal{F}_{t}\right)_{t \geq 0}, \mathbb{P}\right)$. We also assume that $L, L_{0}$ and $W_{2}$ are mutually independent.

Let the signal process $X$ be the solution of the following SDE with random initial data $X_{0}$ :

$$
\left\{\begin{array}{l}
d X(t)=b(X(t)) d t+d L_{0}(t)+d L_{1}(t), \quad t>0 \\
X(0)=X_{0}
\end{array}\right.
$$

Here $b: \mathbb{R} \rightarrow \mathbb{R}$ is a Lipschitz continuous function. Also we suppose that the observable process $Y$ solves the following SDE with random initial data $Y_{0}$.

$$
\left\{\begin{array}{l}
d Y(t)=g(X(t)) d t+d L_{2}(t)+d W_{2}(t), \quad t>0 \\
Y(0)=Y_{0}
\end{array}\right.
$$

where $g: \mathbb{R} \rightarrow \mathbb{R}$ is a twice differentiable mapping. Let $\left\{\mathcal{X}_{t}: t \geq 0\right\}$ and $\left\{\mathcal{Y}_{t}: t \geq\right.$ $0\}$ be the filtration defined by $\mathcal{X}_{t}=\sigma(\{X(s), s \leq t\})$ and $\mathcal{Y}_{t}=\sigma(\{Y(s), s \leq t\})$, respectively. In addition, let $\mathcal{X}=\left(\bigcup_{t \geq 0} \mathcal{X}_{t}\right)$ and $\mathcal{Y}=\left(\bigcup_{t \geq 0} \mathcal{Y}_{t}\right)$.

The filtering problem consists of determining at a fixed time $t>0$ the conditional distribution $\pi_{t}$ of the signal $X$ given the information accumulated from observing $Y$ in the time interval $[0, t]$; that is, for $f \in C_{b}^{(2)}(\mathbb{R})$, we are aiming to compute the Bayes estimator

$$
\pi_{t}(f)=\mathbb{E}\left[f(X(t)) \mid \mathcal{Y}_{t}\right], \quad t \geq 0 .
$$


In order to study about the normalized conditional density $\pi=\left\{\pi_{t}: t \geq 0\right\}$, one can mainly use two different methods. The first one is probability measure transformation and obtain Zakai equation which solves the un-normalized conditional density associated with normalized density $\pi$. Then discuss about $\pi$ using Kallianpur-Striebel formula (see Bain and Crisan (2009), Proposition 3.16). The second method is called innovation approach which directly gives FujisakiKallianpur-Kunita equation (called "FKK equation"). Normalized density $\pi$ is the solution of FKK equation. In this paper, we use the former method.

In the first step, we apply the Girsanov's theorem to get a new measure $\mathbb{Q}$ which is chosen in such a way that $Y$ is a Lévy process over the probability space $\left(\Omega, \mathcal{Y},\left(\mathcal{Y}_{t}\right)_{t \geq 0}, \mathbb{Q}\right)$. For this purpose, let $Z=\{Z(t): t \geq 0\}$ be given by

$$
Z(t):=\exp \left(-\int_{0}^{t} g(X(s)) d W_{2}(s)-\frac{1}{2} \int_{0}^{t} g^{2}(X(s)) d s\right), \quad t \geq 0 .
$$

Note, that $Z$ solves

$$
\left\{\begin{array}{l}
d Z(t)=Z\left(t^{-}\right) g\left(X\left(t^{-}\right)\right) d W_{2}(t) \\
Z(0)=1
\end{array}\right.
$$

$\operatorname{over}\left(\Omega, \mathcal{F},\left(\mathcal{F}_{t}\right)_{t \geq 0}, \mathbb{P}\right)$. Let $\mathbb{Q}$ be a new probability measure given by

$$
\left.\frac{d \mathbb{Q}}{d \mathbb{P}}\right|_{\mathcal{F}_{t}}=Z(t), \quad t \geq 0
$$

As in the Brownian case, one can show the following proposition.

Proposition 2.1. If

$$
\mathbb{E}\left[\int_{0}^{t}\|g(X(s))\|^{2} d s\right]<\infty, \quad \mathbb{E}\left[\int_{0}^{t} Z(s)\|g(X(s))\| d s\right]<\infty, \quad t \geq 0,
$$

then under $\mathbb{Q}$ the observation process $Y$ is a Lévy process. In particular, the $\sigma$-field $\mathcal{Y}_{t}^{+}=\sigma(Y(r)-Y(s), t \leq s \leq r)$ is independent to $\mathcal{Y}_{t}$.

Proof. Let $\mathbb{Q}$ be defined as in equation (2.7). First, note that by the Itô-Lévy decomposition the continuous and discontinuous parts of $Y$ are independent. In addition, under the new probability measure $\mathbb{Q}$, the continuous part of $Y$ is a Brownian motion. We can also see that the pure jump process is not affected by the change of measure.

Setting $V(t)=Z(t)^{-1}$, we obtain as in Bain and Crisan (2009), Eq. (3.30), page 56 , that

$$
\left.\frac{d \mathbb{P}}{d \mathbb{Q}}\right|_{\mathcal{F}_{t}}=V(t), \quad t \geq 0
$$


Remark 2.1. The process $V=\{V(t): t \geq 0\}$ defined by $V(t)=Z(t)^{-1}$ solves on $(\Omega, \mathcal{F}, \mathbb{P})$ the equation

$$
\left\{\begin{aligned}
d V(t) & =V(t) g(X(t))\left[d W_{2}(t)+g(X(t)) d t\right] \\
& =V(t) g(X(t)) d Y^{c}(t) \\
V(0) & =1
\end{aligned}\right.
$$

( $Y^{c}$ denotes the continuous part of $Y$, i.e. the part of $Y$ without jumps.) Since the process $W_{2}(t)+\int_{0}^{t} g(X(s)) d s$ becomes a Brownian motion over $\left(\Omega, \mathcal{F},\left(\mathcal{F}_{t}\right)_{t \geq 0}, \mathbb{Q}\right), V$ is a $\left(\Omega, \mathcal{F},\left(\mathcal{F}_{t}\right)_{t \geq 0}, \mathbb{Q}\right)$-martingale.

The following result is an immediate consequence of Proposition 2.1. We also refer to Bain and Crisan (2009), Proposition 3.15, page 56.

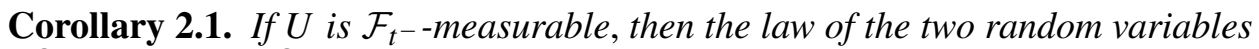
$\mathbb{E}^{\mathbb{Q}}[U \mid \mathcal{Y}]$ and $\mathbb{E}^{\mathbb{Q}}\left[U \mid \mathcal{Y}_{t^{-}}\right]$are the same over $(\Omega, \mathcal{F}, \mathbb{Q})$. In particular, we have $\mathbb{Q}$-a.s.

$$
\mathbb{E}^{\mathbb{Q}}[U \mid \mathcal{Y}]=\mathbb{E}^{\mathbb{Q}}\left[U \mid \mathcal{Y}_{t^{-}}\right]
$$

Remark 2.2. Similarly it can be shown that if $U$ is $\mathcal{F}_{t}$-measurable, then $\mathbb{Q}$-a.s.

$$
\mathbb{E}^{\mathbb{Q}}[U \mid \mathcal{Y}]=\mathbb{E}^{\mathbb{Q}}\left[U \mid \mathcal{Y}_{t}\right]
$$

Proof of Corollary 2.1. Since $Y$ is a Lévy process over $\left(\Omega, \mathcal{F},\left(\mathcal{F}_{t}\right)_{t \geq 0}, \mathbb{Q}\right)$, its increments are independent. Hence, for all $t>0$, the $\sigma$-algebra $\mathcal{Y}_{t^{-}}^{+}$generated by $Y(s)-Y\left(t^{-}\right), s>t$ is independent to $\mathcal{Y}_{t^{-}}$under the measure $\mathbb{Q}$. From Kallenberg (2002), Proposition 6.6, page 110, the assertion follows.

Fix $t \geq 0$. Let $\pi_{t}$ be the conditional distribution of $X(t)$ at time $t \geq 0$. The Kallianpur-Striebel formula gives for $t \geq 0$ (see Bain and Crisan (2009), Proposition 3.16)

$$
\pi_{t}(f)=\mathbb{E}\left[f(X(t)) \mid \mathcal{Y}_{t}\right]=\int_{\mathbb{R}} \pi_{t}(x) f(x) d x=\frac{\mathbb{E}^{\mathbb{Q}}\left[f(X(t)) V(t) \mid \mathcal{Y}_{t}\right]}{\mathbb{E}^{\mathbb{Q}}\left[V(t) \mid \mathcal{Y}_{t}\right]}
$$

Now, we introduce the density process of the un-normalized conditional distribution $\rho=\left\{\rho_{t}: t \geq 0\right\}$ which is the measure valued process defined by

$$
\begin{aligned}
\rho_{t}(f) & =\left\langle\rho_{t}, f\right\rangle=\mathbb{E}^{\mathbb{Q}}\left[V(t) f(X(t)) \mid \mathcal{Y}_{t}\right] \\
& =\int_{\mathbb{R}} \rho_{t}(x) f(x) d x, \quad t>0, \rho_{0}=\pi_{0} .
\end{aligned}
$$

We will see later on, that the process $\rho=\left\{\rho_{t}: t \geq 0\right\}$ is very useful to calculate $\pi=\left\{\pi_{t}: t \geq 0\right\}$. 
By Corollary 2.1, we have

$$
\mathbb{E}^{\mathbb{Q}}[f(X(t)) V(t) \mid \mathcal{Y}]=\mathbb{E}^{\mathbb{Q}}\left[f(X(t)) V(t) \mid \mathcal{Y}_{t}\right]=\left\langle\rho_{t}, f\right\rangle, \quad t \geq 0, \mathbb{Q} \text {-a.s. }
$$

We also introduce the process $\xi=\{\xi(t): t \geq 0\}$ defined by

$$
\xi(t)=\mathbb{E}^{\mathbb{Q}}\left[V(t) \mid \mathcal{Y}_{t}\right], \quad t \geq 0
$$

Since $V$ is a $\mathcal{F}_{t}$-martingale over $(\Omega, \mathcal{F}, \mathbb{Q})$ and $\mathcal{Y}_{t} \subset \mathcal{F}_{t}$, it follows that for $0 \leq$ $s<t$

$$
\mathbb{E}^{\mathbb{Q}}\left[\xi(t) \mid \mathcal{Y}_{s}\right]=\mathbb{E}^{\mathbb{Q}}\left[\mathbb{E}^{\mathbb{Q}}\left[V(t) \mid \mathcal{F}_{s}\right] \mid \mathcal{Y}_{s}\right]=\mathbb{E}^{\mathbb{Q}}\left[V(s) \mid \mathcal{Y}_{s}\right]=\xi(s) .
$$

Moreover,

$$
\xi(t) \pi_{t}(f)=\rho_{t}(f), \quad t \geq 0
$$

and

$$
\pi_{t}(f)=\rho_{t}(f) \xi^{-1}(t), \quad t \geq 0 .
$$

For these two formulas, we refer to Bain and Crisan (2009), Definition 3.17 and Corollary 3.19, pages 58-59.

In the next theorem, we will derive the Zakai equation which is solved by the un-normalized density process $\rho=\left\{\rho_{t}: t \geq 0\right\}$. To do that, we need to introduce some additional notations. A Lévy process $L$ is characterized by its characteristic function. In particular, there exists a function $\psi: \mathbb{R} \mapsto \mathbb{C}$ such that

$$
\ln \left(\mathbb{E} e^{i \xi L(t)}\right)=t \psi(\xi), \quad \xi \in \mathbb{R} .
$$

The infinitesimal generator of the Markovian semigroup of $L$ is the so called pseudo-differential operator given by

$$
A_{0} f:=-\int_{\mathbb{R}} e^{i \xi x} \psi(\xi) \mathcal{F} f(\xi) d \xi, \quad f \in C_{b}^{(2)}(\mathbb{R}) .
$$

Here $\mathcal{F} f$ denotes the Fourier transform of the function $f$. The function $\psi$ is called the Lévy symbol of the Lévy process $X$, for more details on $A_{0}$ and its properties we refer to Fernando Pani, Hausenblas and Razafimandimby (2017). The following theorem associates with the case where the Lévy measure of the two dimensional Lévy process $L$ is finite.

Theorem 2.2. Let $L_{0}$ be a Lévy process with Lévy symbol $\psi$ and $A_{0}$ be the infinitesimal generator of $L_{0}$. Let $v_{1}$ and $v_{2}$ be two finite Lévy measures defined on the positive half real line, that is, on $\mathbb{R}_{+}$. Let $H$ be a twice differentiable copula. Let us denote the conditional Lévy measure of jumps of $L_{1}$ given the jumps of $L_{2}$ by

$$
v_{1, z_{2}}(U)=\int_{U} h\left(z_{1}, z_{2}\right) v_{1}\left(d z_{1}\right), \quad U \in \mathcal{B}\left(\mathbb{R}_{+}\right)
$$


where

$$
h\left(z_{1}, z_{2}\right):=\left.\frac{\partial^{2}}{\partial u_{1} \partial u_{2}} H\left(u_{1}, u_{2}\right)\right|_{\substack{u_{1}=U_{1}\left(z_{1}\right) \\ u_{2}=U_{2}\left(z_{2}\right)}},
$$

and $U_{1}, U_{2}$ are the tail integrals of $v_{1}$ and $v_{2}$, respectively. Let $g: \mathbb{R} \rightarrow \mathbb{R}$ and $\sigma: \mathbb{R} \rightarrow \mathbb{R}$ be Lipschitz continuous mappings. Then the un-normalized conditional density estimator $\rho=\left\{\rho_{t}: t \geq 0\right\}$ is a solution to the following equation

$$
\begin{aligned}
\left\langle\rho_{t}, f\right\rangle= & \left\langle\rho_{0}, f\right\rangle+\int_{0}^{t}\left\langle\rho_{s^{-}}, f \cdot g\right\rangle d Y_{s}^{c}+\int_{0}^{t}\left\langle\rho_{s^{-}}, \mathcal{A}_{0} f\right\rangle d s \\
& +\int_{0}^{t}\left\langle\rho_{s^{-}}, \Theta_{z_{2}} f\right\rangle \eta_{2}\left(d z_{2}, d s\right), \quad \forall f \in C_{b}^{(2)}(\mathbb{R}),
\end{aligned}
$$

where $\eta_{2}$ denotes the Poisson random measure associated to $L_{2}$ with intensity $v_{2}$, the operators $\Theta_{z}$ and $\mathcal{A}_{0}$ are defined by

$$
\Theta_{z} f(x)=\int_{\mathbb{R}_{+}}\left[f\left(x+z_{1}\right)-f(x)\right] v_{1, z}\left(d z_{1}\right), \quad z \in \mathbb{R}_{+}, x \in \mathbb{R}, f \in C_{b}^{(2)}(\mathbb{R}),
$$

and

$$
\mathcal{A}_{0} f(x)=b(x) f^{\prime}(x)+A_{0} f(x), \quad x \in \mathbb{R}, f \in C_{b}^{(2)}(\mathbb{R}),
$$

where the operator $A_{0}$ is the infinitesimal generator of the Markovian semigroup of $L_{0}$ which is a pseudo-differential operator and defined through (2.10).

Remark 2.3. Since $v_{1}$ and $v_{2}$ are finite Lévy measures, the operator $\Theta_{z}$ : $H_{2}^{s}(\mathbb{R}) \rightarrow H_{2}^{s}(\mathbb{R})$ is bounded for all $z \in \mathbb{R}$ and $s \in \mathbb{R}$. This can be seen by analyzing the symbol $\phi_{z}$ associated to $\Theta_{z}$ defined as

$$
\phi_{z}(\xi)=\int_{\mathbb{R}_{+}}\left(e^{i z_{1} \xi}-1\right) h\left(z_{1}, z\right) v_{1}\left(d z_{1}\right) .
$$

In fact, calculating the modulus of the symbol $\phi_{z}$

$$
\left|\phi_{z}(\xi)\right|:=\left|\int_{\mathbb{R}_{+}}\left(e^{i z_{1} \xi}-1\right) h\left(z_{1}, z\right) v_{1}\left(d z_{1}\right)\right| \leq 2 \int_{\mathbb{R}_{+}}\left|h\left(z_{1}, z\right)\right| v_{1}\left(d z_{1}\right)<\infty,
$$

we see that $\left|\phi_{z}(\xi)\right| \leq C$ for all $\xi \in \mathbb{R}$. Therefore, $\Phi_{z}: L^{2}(\mathbb{R}) \rightarrow L^{2}(\mathbb{R})$ defined by

$$
\left(\Phi_{z} u\right)(\xi):=\phi_{z}(\xi) u(\xi), \quad \xi \in \mathbb{R}, u \in L^{2}(\mathbb{R}),
$$

is a bounded operator. Using the spectral theorem (see, e.g., Engel and Nagel (2000), Theorem 4.9, page 30) one sees, that $\Phi_{z}$ acting on $L^{2}(\mathbb{R})$ as a multiplication operator corresponds via the Fourier transform to $\Theta_{z}$ acting on $L^{2}(\mathbb{R})$. Next, the operator $\mathcal{F}^{-1}\left(1+|\xi|^{2}\right)^{\frac{s}{2}} \mathcal{F}$ is an isometry from $H_{2}^{s}(\mathbb{R})$ to $L^{2}(\mathbb{R})$. Hence, $\Phi_{z}$ is also bounded on $H_{2}^{s}(\mathbb{R})$. This implies that $\Theta_{z}: H_{2}^{s}(\mathbb{R}) \rightarrow H_{2}^{s}(\mathbb{R})$ is bounded for all $z \in \mathbb{R}$ and $s \in \mathbb{R}$. 
Proof of Theorem 2.2. Let $\lambda_{1}=v_{1}\left(\mathbb{R}_{+}\right)$and $\lambda_{2}=v_{2}\left(\mathbb{R}_{+}\right)$. Next, let us denote the number of jumps of $L_{2}$ in the time interval $[0, t]$ by $N(t)$, the jumps themselves by $\left\{Y_{2, i}: i=1, \ldots, N(t)\right\}$ and the jump times by $\left\{s_{i}: i=1, \ldots, N(t)\right\}$. Then, given the jumps of $L_{2}$ in the time interval $[0, t], L_{1}(t)$ can be represented by

$$
L_{1}(t)=\sum_{i=1}^{N(t)} Y_{Y_{2, i}}^{1, i}, \quad t \geq 0,
$$

where for $z \in \mathbb{R} \backslash\{0\}$ the random variable $Y_{z}^{1}$ is distributed as $\nu_{1, z} / \lambda_{1, z}, \lambda_{1, z}=$ $v_{1, z}\left(\mathbb{R}^{+}\right)$. More rigorously, conditioned on the jumps of $L_{2}(t), L_{1}(t)$ can be viewed as a compound Poisson process having same jump times of $L_{2}(t)$ and the size of each jump $Y^{1, i}$ of $L_{1}(t)$ depends on the size of the jump $Y_{2, i}$ at time $s_{i}$.

By conditioning the process $L_{1}$ given $L_{2}$, we can write

$$
\begin{aligned}
& f(X(t))=f\left(X_{0}\right)+\int_{0}^{t}\left(\mathcal{A}_{0} f\right)(X(s)) d s+M(t) \\
& +\sum_{1 \leq i \leq N(t)} f\left(X\left(s_{i}^{-}\right)+Y_{Y_{2, i}}^{1, i}\right)-f\left(X\left(s_{i}^{-}\right)\right) \\
& =f\left(X_{0}\right)+\int_{0}^{t}\left(\mathcal{A}_{0} f\right)(X(s)) d s+M(t) \\
& +\int_{0}^{t} \int_{\mathbb{R}_{0}^{+}} \int_{\mathbb{R}_{0}^{+}}\left[f\left(X\left(s^{-}\right)+z_{1}\right)-f\left(X\left(s^{-}\right)\right)\right] \\
& \times v_{1, z_{2}}\left(d z_{1}\right) \eta_{2}\left(d z_{2}, d s\right) \\
& +\sum_{1 \leq i \leq N(t)} f\left(X\left(s_{i}^{-}\right)+Y_{Y_{2, i}}^{1, i}\right)-f\left(X\left(s_{i}^{-}\right)\right) \\
& -\int_{0}^{t} \int_{\mathbb{R}_{0}^{+}} \int_{\mathbb{R}_{0}^{+}}\left[f\left(X\left(s^{-}\right)+z_{1}\right)-f\left(X\left(s^{-}\right)\right)\right] \\
& \times v_{1, z_{2}}\left(d z_{1}\right) \eta_{2}\left(d z_{2}, d s\right) \\
& =f\left(X_{0}\right)+\int_{0}^{t}\left(\mathcal{A}_{0} f\right)(X(s)) d s+M(t)+\tilde{M}(t) \\
& +\int_{0}^{t} \int_{\mathbb{R}_{0}^{+}} \int_{\mathbb{R}_{0}^{+}}\left[f\left(X\left(s^{-}\right)+z_{1}\right)-f\left(X\left(s^{-}\right)\right)\right] \\
& \times v_{1, z_{2}}\left(d z_{1}\right) \eta_{2}\left(d z_{2}, d s\right),
\end{aligned}
$$

where

$$
M(t)=\int_{0}^{t} f^{\prime}(X(s)) d L_{0}(s), \quad t \geq 0
$$


and

$$
\begin{aligned}
\tilde{M}(t)= & J(t)-R(t) \\
= & \sum_{1 \leq i \leq N(t)} f\left(X\left(s_{i}^{-}\right)+Y_{Y_{2, i}}^{1, i}\right)-f\left(X\left(s_{i}^{-}\right)\right) \\
& -\int_{0}^{t} \int_{\mathbb{R}_{0}^{+}} \int_{\mathbb{R}_{0}^{+}}\left[f\left(X\left(s^{-}\right)+z_{1}\right)-f\left(X\left(s^{-}\right)\right)\right] \\
& \times v_{1, z_{2}}\left(d z_{1}\right) \eta_{2}\left(d z_{2}, d s\right), \quad t \geq 0 .
\end{aligned}
$$

Since $L_{0}$ be a compensated pure jump Lévy process, the process $M=\{M(t): t \geq$ $0\}$ is a martingale over $\left(\Omega, \mathcal{F},\left(\mathcal{F}_{t}\right)_{t \geq 0}, \mathbb{Q}\right)$. First, observe that we can write for a function $\phi$

$$
\sum_{i=1}^{N(t)} \phi\left(Y_{2, i}\right)=\sum_{i=1}^{N(t)} \int_{\mathbb{R}^{+}} \phi\left(z_{2}\right) \eta_{2}\left(d z_{2},\left\{s_{i}\right\}\right) .
$$

In addition, we have by the tower property

$$
\begin{aligned}
\mathbb{E}[J(t) \mid k & \left.=N(t),\left(z_{2,1}, \ldots, z_{2, k}\right)=\left(Y_{2,1}, \ldots, Y_{2, k}\right)\right] \\
= & \mathbb{E}\left[\sum_{1 \leq i \leq k} f\left(X\left(s_{i}^{-}\right)+Y_{Y_{2, i}}^{1, i}\right)-f\left(X\left(s_{i}^{-}\right)\right) \mid\right. \\
& \left.k=N(t),\left(z_{2,1}, \ldots, z_{2, k}\right)=\left(Y_{2,1}, \ldots, Y_{2, k}\right)\right] \\
= & \mathbb{E}\left[\sum_{1 \leq i \leq k} \mathbb{E}\left[f\left(X\left(s_{i}^{-}\right)+Y_{Y_{2, i}}^{1, i}\right)-f\left(X\left(s_{i}^{-}\right)\right) \mid Y_{2, i}=z_{2, i}\right] \mid k=N(t)\right] \\
= & \mathbb{E}\left[\sum_{1 \leq i \leq k} \int_{\mathbb{R}^{+}}\left\{f\left(X\left(s_{i}^{-}\right)+z_{1}\right)-f\left(X\left(s_{i}^{-}\right)\right)\right\} v_{1, z_{2, i}}\left(d z_{1}\right) \mid k=N(t)\right] .
\end{aligned}
$$

Using the representation above, we get

$$
\cdots=\sum_{i=1}^{N(t)} \int_{\mathbb{R}^{+}} \int_{\mathbb{R}^{+}}\left\{f\left(X\left(s_{i}^{-}\right)+z_{1}\right)-f\left(X\left(s_{i}^{-}\right)\right)\right\} v_{1, z_{2}}\left(d z_{1}\right) \eta_{2}\left(d z_{2},\left\{s_{i}\right\}\right) .
$$

Replacing the summation by the integral with respect to the time, we get

$$
\cdots=\int_{0}^{t} \int_{\mathbb{R}_{0}^{+}} \int_{\mathbb{R}_{0}^{+}}\left[f\left(X\left(s^{-}\right)+z_{1}\right)-f\left(X\left(s^{-}\right)\right)\right] v_{1, z_{2}}\left(d z_{1}\right) \eta_{2}\left(d z_{2}, d s\right)=R(t) .
$$

Now we want to show that $\mathbb{E}^{\mathbb{Q}}\left[\tilde{M}(t) \mid \mathcal{Y}_{t}\right]=0, t \geq 0$. Fix $t \geq 0$. Then

$$
\begin{aligned}
\mathbb{E}^{\mathbb{Q}}\left[\tilde{M}(t) \mid \mathcal{Y}_{t}\right] & =\mathbb{E}^{\mathbb{Q}}\left[J(t)-R(t) \mid \mathcal{Y}_{t}\right] \\
& =\mathbb{E}^{\mathbb{Q}}\left[J(t) \mid \mathcal{Y}_{t}\right]-\mathbb{E}^{\mathbb{Q}}\left[R(t) \mid \mathcal{Y}_{t}\right]
\end{aligned}
$$




$$
\begin{aligned}
& =\mathbb{E}^{\mathbb{Q}}\left[\mathbb{E}^{\mathbb{Q}}\left[J(t) \mid \mathcal{F}_{1}\right] \mid \mathcal{Y}_{t}\right]-\mathbb{E}^{\mathbb{Q}}\left[R(t) \mid \mathcal{Y}_{t}\right] \\
& =\mathbb{E}^{\mathbb{Q}}\left[R(t) \mid \mathcal{Y}_{t}\right]-\mathbb{E}^{\mathbb{Q}}\left[R(t) \mid \mathcal{Y}_{t}\right]=0,
\end{aligned}
$$

where $\mathcal{F}_{1}=\left\{k=N(t),\left(z_{2,1}, \ldots, z_{2, k}\right)=\left(Y_{2,1}, \ldots, Y_{2, k}\right): k \in \mathbb{N}\right\} \subseteq \mathcal{Y}_{t}$. Under the new probability measure $\mathbb{Q}$, the process $V=\{V(t): t \geq 0\}$ solves the following SDE

$$
d V(t)=V(t) g(X(t)) d Y^{c}(t), \quad t>0, V(0)=1,
$$

where $Y^{c}$ denotes the continuous part of $Y$ which is a Brownian motion under $\mathbb{Q}$, adapted to $\left(\mathcal{Y}_{t}\right)_{t \geq 0}$. Since $V$ is driven by the continuous part of $Y$, and $L_{0}$ independent from $W_{2}$, no correlation terms involving the process $V$ appears. Thus, we get

$$
\begin{aligned}
f(X(t)) V(t)= & f\left(X_{0}\right)+\int_{0}^{t} V\left(s^{-}\right) d M(s)+\int_{0}^{t} V\left(s^{-}\right) d \tilde{M}(s) \\
& +\int_{0}^{t} \int_{\mathbb{R}_{0}^{+}} V\left(s^{-}\right) \int_{\mathbb{R}_{0}^{+}}\left[f\left(X\left(s^{-}\right)+z_{1}\right)-f\left(X\left(s^{-}\right)\right)\right] \\
& \times v_{1, z_{2}}\left(d z_{1}\right) \eta_{2}\left(d z_{2}, d s\right) \\
& +\int_{0}^{t} V(s) g(X(s)) f(X(s)) d Y^{c}(s)+\int_{0}^{t} V(s)\left(\mathcal{A}_{0} f\right)(X(s)) d s .
\end{aligned}
$$

Taking into account that $M$ is a martingales over $\left(\Omega, \mathcal{F},\left(\mathcal{Y}_{t}\right)_{t \geq 0}, \mathbb{Q}\right)$ with $(2.14)$ and taking conditional expectation together with the Fubini theorem Applebaum (2009), Theorem 1.1.8, to the entity above, we get

$$
\begin{aligned}
\mathbb{E}^{\mathbb{Q}}\left[f(X(t)) V(t) \mid \mathcal{Y}_{t}\right]= & \mathbb{E}^{\mathbb{Q}}\left[f\left(X_{0}\right) \mid \mathcal{Y}_{0}\right]+\underbrace{\mathbb{E}^{\mathbb{Q}}\left[\int_{0}^{t} V\left(s^{-}\right) d M(s) \mid \mathcal{Y}_{t}\right]}_{=0} \\
& +\underbrace{\mathbb{E}\left[\int_{0}^{t} V\left(s^{-}\right) d \tilde{M}(s) \mid \mathcal{Y}_{t}\right]} \\
& +\mathbb{E}^{\mathbb{Q}}\left[\int_{0}^{t} V(s)\left(\mathcal{A}_{0} f\right)(X(s)) d s \mid \mathcal{Y}_{t}\right] \\
& +\mathbb{E}^{\mathbb{Q}}\left[\int_{0}^{t} V(s) g(X(s)) f(X(s)) d Y_{s}^{c} \mid \mathcal{Y}_{t}\right] \\
& +\mathbb{E}^{\mathbb{Q}}\left[\int_{0}^{t} \int_{\mathbb{R}_{0}^{+}} V\left(s^{-}\right) \int_{\mathbb{R}_{0}^{+}}\left[f\left(X\left(s^{-}\right)+z_{1}\right)-f\left(X\left(s^{-}\right)\right)\right]\right. \\
& \left.\times v_{1, z_{2}}\left(d z_{1}\right) \eta_{2}\left(d z_{2}, d s\right) \mid \mathcal{Y}_{t}\right] \\
= & f\left(X_{0}\right)+\int_{0}^{t} \mathbb{E}^{\mathbb{Q}}\left[V(s)\left(\mathcal{A}_{0} f\right)(X(s)) \mid \mathcal{Y}_{t}\right] d s
\end{aligned}
$$




$$
\begin{aligned}
& +\mathbb{E}^{\mathbb{Q}}\left[\int_{0}^{t} \int_{\mathbb{R}_{0}^{+}} V\left(s^{-}\right) \int_{\mathbb{R}_{0}^{+}}\left[f\left(X\left(s^{-}\right)+z_{1}\right)-f\left(X\left(s^{-}\right)\right)\right]\right. \\
& \left.\times v_{1, z_{2}}\left(d z_{1}\right) \eta_{2}\left(d z_{2}, d s\right) \mid \mathcal{Y}_{t}\right] \\
& +\mathbb{E}^{\mathbb{Q}}\left[\int_{0}^{t} V(s) g(X(s)) f(X(s)) d Y_{s}^{c} \mid \mathcal{Y}_{t}\right]
\end{aligned}
$$

By imitating the calculations associated with (2.13) and (2.14) for

$$
\int_{0}^{t} V\left(s^{-}\right) d \tilde{M}(s)=\int_{0}^{t} V\left(s^{-}\right) d J(s)-\int_{0}^{t} V\left(s^{-}\right) d R(s),
$$

we could show that

$$
\mathrm{E}^{\mathrm{Q}}\left[\int_{0}^{t} V\left(s^{-}\right) d \tilde{M}(s) \mid \mathcal{Y}_{t}\right]=0 .
$$

In the next step, we show that

$$
\mathbb{E}^{\mathbb{Q}}\left[\int_{0}^{t} V\left(s^{-}\right) d M(s) \mid \mathcal{Y}_{t}\right]=0
$$

Since $\int_{0}^{t} V\left(s^{-}\right) d M(s)$ is $\mathcal{F}_{t}$-measurable, it follows from Remark 2.2

$$
\begin{aligned}
\mathbb{E}^{\mathbb{Q}}\left[\int_{0}^{t} V\left(s^{-}\right) d M(s) \mid \mathcal{Y}_{t}\right] & =\mathbb{E}^{\mathbb{Q}}\left[\int_{0}^{t} V\left(s^{-}\right) f^{\prime}\left(X\left(s^{-}\right)\right) d L_{0}(s) \mid \mathcal{Y}_{t}\right] \\
& =\mathbb{E}^{\mathbb{Q}}\left[\int_{0}^{t} V\left(s^{-}\right) f^{\prime}\left(X\left(s^{-}\right)\right) d L_{0}(s) \mid \mathcal{Y}\right] .
\end{aligned}
$$

By following to Bain and Crisan (2009), page 60, the proof of the part (ii) of Lemma 3.21, similar arguments, we get

$$
\begin{aligned}
\mathbb{E}^{\mathbb{Q}}\left[\varepsilon_{t} \mathbb{E}^{\mathbb{Q}}\left[\int_{0}^{t} V\left(s^{-}\right) f^{\prime}\left(X\left(s^{-}\right)\right) d L_{0}(s) \mid \mathcal{Y}\right]\right] \\
=\mathbb{E}^{\mathbb{Q}}\left[\varepsilon_{t} \int_{0}^{t} V\left(s^{-}\right) f^{\prime}\left(X\left(s^{-}\right)\right) d L_{0}(s)\right] \\
=\mathbb{E}^{\mathbb{Q}}\left[\int_{0}^{t} V\left(s^{-}\right) f^{\prime}\left(X\left(s^{-}\right)\right) d L_{0}(s)\right] \\
+\mathbb{E}^{\mathbb{Q}}\left\langle\int_{0}^{t} i \varepsilon_{s} r_{s} d Y^{c}(s), \int_{0}^{\cdot} V\left(s^{-}\right) f^{\prime}\left(X\left(s^{-}\right)\right) d L_{0}(s)\right\rangle_{t} \\
=\mathbb{E}^{\mathbb{Q}}\left[\int_{0}^{t} V\left(s^{-}\right) f^{\prime}\left(X\left(s^{-}\right)\right) d L_{0}(s)\right] \\
+\mathbb{E}^{\mathbb{Q}} \int_{0}^{t} i \varepsilon_{s} r_{s} \int_{0}^{\cdot} V\left(s^{-}\right) f^{\prime}\left(X\left(s^{-}\right)\right)\left\langle d Y^{c}(s), d L_{0}(s)\right\rangle_{t}=0,
\end{aligned}
$$


where $\varepsilon_{t}=1+\int_{0}^{t} i \varepsilon_{s} r_{s} d Y^{c}(s)$ is a member of the total set define in Bain and Crisan (2009), page 355, (B.19). This implies that $\mathbb{E}^{\mathbb{Q}}\left[\int_{0}^{t} V\left(s^{-}\right) d M(s) \mid \mathcal{Y}_{t}\right]=0$ for any $s \in[0, t]$. Since $X(s)$ and $V(s)$ are $\mathcal{F}_{s}$-measurable, we have

$$
\mathbb{E}^{\mathbb{Q}}\left[V(s)\left(\mathcal{A}_{0} f\right)(X(s)) \mid \mathcal{Y}_{t}\right]=\mathbb{E}^{\mathbb{Q}}\left[V(s)\left(\mathcal{A}_{0} f\right)(X(s)) \mid \mathcal{Y}_{s}\right]
$$

Since $Y^{c}(t)$ is $\mathcal{Y}_{t}$-measurable and is a $\mathbb{Q}$-Brownian motion, it follows from Borkar (1989), Lemma 1.2, and Corollary 2.1

$$
\begin{aligned}
\mathbb{E}^{\mathbb{Q}}\left[\int_{0}^{t} V(s) g(X(s)) f(X(s)) d Y_{s}^{c} \mid \mathcal{Y}_{t}\right] \\
\quad=\int_{0}^{t} \mathbb{E}^{\mathbb{Q}}\left[V(s) g(X(s)) f(X(s)) \mid \mathcal{Y}_{t}\right] d Y_{s}^{c} \\
\quad=\int_{0}^{t} \mathbb{E}^{\mathbb{Q}}\left[V(s) g(X(s)) f(X(s)) \mid \mathcal{Y}_{s}\right] d Y_{s}^{c} .
\end{aligned}
$$

Due to the fact that $V\left(s^{-}\right) \int_{\mathbb{R}_{0}^{+}}\left[f\left(X\left(s^{-}\right)+y\right)-f\left(X\left(s^{-}\right)\right)\right] v_{1, z_{2}}(d y)$ is a $\mathcal{F}_{s^{--}}$ measurable random variable and $\Delta L_{2}=L_{2}(s)-L_{2}\left(s^{-}\right)$is independent from $\mathcal{F}_{s^{-}}$, it follows again by Corollary 2.1

$$
\begin{aligned}
\mathbb{E}^{\mathbb{Q}}\left[\int_{0}^{t} \int_{\mathbb{R}_{0}^{+}} V\left(s^{-}\right) \int_{\mathbb{R}_{0}^{+}}\left[f\left(X\left(s^{-}\right)+z_{1}\right)-f\left(X\left(s^{-}\right)\right)\right] v_{1, z_{2}}\left(d z_{1}\right) \eta_{2}\left(d z_{2}, d s\right) \mid \mathcal{Y}_{t}\right] \\
=\int_{0}^{t} \int_{\mathbb{R}_{0}^{+}} \mathbb{E}^{\mathbb{Q}}\left[V\left(s^{-}\right) \int_{\mathbb{R}_{0}^{+}}\left[f\left(X\left(s^{-}\right)+z_{1}\right)-f\left(X\left(s^{-}\right)\right)\right] v_{1, z_{2}}\left(d z_{1}\right) \mid \mathcal{Y}_{t}\right] \\
\quad \times \eta_{2}\left(d z_{2}, d s\right) \\
=\int_{0}^{t} \int_{\mathbb{R}_{0}^{+}} \int_{\mathbb{R}_{0}^{+}} \mathbb{E}^{\mathbb{Q}}\left[V\left(s^{-}\right)\left[f\left(X\left(s^{-}\right)+z_{1}\right)-f\left(X\left(s^{-}\right)\right)\right] v_{1, z_{2}}\left(d z_{1}\right) \mid \mathcal{Y}_{s^{-}}\right] \\
\quad \times \eta_{2}\left(d z_{2}, d s\right) .
\end{aligned}
$$

By collecting all the results, one can conclude the theorem.

In the case where the Lévy measure of $L$ is $\sigma$-finite, the copula has to satisfy certain scaling properties. Namely, we have to take $H$ such that

$$
\lim _{\gamma \rightarrow \infty} \frac{H(\gamma u, \gamma v)}{H(\gamma, \gamma)}=H(u, v), \quad u, v \in \mathbb{R} .
$$

Now we can formulate the following theorem for the case where Lévy measure of $L$ is $\sigma$-finite.

Theorem 2.3. Let $L_{0}$ be a Lévy process with symbol $\psi$. Let $v_{1}$ and $v_{2}$ be two $\sigma$-finite Lévy measures such that

$$
\int_{|z| \leq 1}|z| v_{1}(d z)+\int_{|z| \leq 1}|z| v_{2}(d z)<\infty .
$$


Let $H$ be a twice differentiable copula which satisfies the scaling property (2.18). Let

$$
v_{1, z_{2}}(U)=\int_{U} h\left(z_{1}, z_{2}\right) v_{1}\left(d z_{1}\right), \quad U \in \mathcal{B}(\mathbb{R} \backslash\{0\}),
$$

where

$$
h\left(z_{1}, z_{2}\right):=\left.\frac{\partial^{2}}{\partial u_{1} \partial u_{2}} H\left(u_{1}, u_{2}\right)\right|_{\substack{u_{1}=U_{1}\left(z_{1}\right) \\ u_{2}=U_{2}\left(z_{2}\right)}},
$$

and $U_{1}, U_{2}$ are the tail integrals of $\nu_{1}$ and $\nu_{2}$, respectively. Let $g, b: \mathbb{R} \rightarrow \mathbb{R}$ and $\sigma$ : $\mathbb{R} \rightarrow \mathbb{R}$ are Lipschitz continuous mappings and $g \in C_{b}^{(2)}(\mathbb{R})$. The un-normalized conditional density $\rho$ is a unique solution to the equation,

$$
\begin{aligned}
\left\langle\rho_{t}, f\right\rangle= & \left\langle\rho_{0}, f\right\rangle+\int_{0}^{t}\left\langle\rho_{s^{-}}, f \cdot g\right\rangle d Y_{s}^{c}+\int_{0}^{t}\left\langle\rho_{s^{-}}, \mathcal{A}_{0} f\right\rangle d s \\
& +\int_{0}^{t} \int_{\mathbb{R}}\left\langle\rho_{s^{-}}, \Theta_{z_{2}} f\right\rangle \eta_{2}\left(d z_{2}, d s\right), \quad \forall f \in C_{b}^{(2)}(\mathbb{R}),
\end{aligned}
$$

where $\eta_{2}$ is the Poisson random measure associated to $L_{2}$ and the operators $\Theta=$ $\left\{\Theta_{z}: z \in \mathbb{R} \backslash\{0\}\right\}, \mathcal{A}_{0}$ are given by

$$
\begin{aligned}
& \Theta_{z} f(x)=\int_{\mathbb{R}}\left[f\left(x+z_{1}\right)-f(x)\right] v_{1, z}\left(d z_{1}\right), \quad x \in \mathbb{R}, z \in \mathbb{R} \backslash\{0\}, \\
& \mathcal{A}_{0} f(x)=b(x) f^{\prime}(x)+A_{0} f(x), \quad x \in \mathbb{R}, f \in C_{b}^{(2)}(\mathbb{R}) .
\end{aligned}
$$

Here $A_{0}$ is the pseudo-differential operator associated with $L_{0}$.

Remark 2.4. By taking $f=1$ in (2.20) and taking into account that $\mathcal{A}_{0} 1=0$, $\Theta_{z} 1=0$, it follows that $\xi$ solves

$$
\begin{aligned}
\xi(t) & =1+\int_{0}^{t} \rho_{s}(g) d Y_{s}^{c}=1+\int_{0}^{t} \rho_{s^{-}}(1) \pi_{s}(g) d Y_{s}^{c} \\
& =1+\int_{0}^{t} \xi(s) \pi_{s}(g) d Y_{s}^{c}, \quad t \geq 0 .
\end{aligned}
$$

Second and third equalities hold due to Kallianpur-Streibel formula and the fact that $\rho_{s}(1)=\xi(s)$ respectively. Hence, the inverse $\varsigma=\{\varsigma(t): t \geq 0\}$ of $\xi$ is given by

$$
\begin{aligned}
\varsigma(t) & =\varsigma(0)+\int_{0}^{t} \varsigma\left(s^{-}\right) \pi_{s}(g)^{2} d s-\int_{0}^{t} \pi_{s}(g) d Y_{s}^{c} \\
& =\varsigma(0)+\int_{0}^{t} \varsigma(s)^{3} \rho_{s}(g)^{2} d s-\int_{0}^{t} \varsigma(s)^{2} \rho_{s}(g) d Y_{s}^{c} .
\end{aligned}
$$

Since $g \in C^{(2)}(\mathbb{R})$, one can easily show that $\rho(g)=\left\{\rho_{t}(g): t \geq 0\right\}$ is bounded by $|g|_{C_{b}}$ and is well defined. Due to this fact and the Novikov condition, we can see that the process $\varsigma$ exists and well defined. 
Proof of Theorem 2.3. To start with the proof, first let us cut off the small jumps from the Lévy process $L$. For any $\varepsilon>0$, let $v_{1}^{\varepsilon}=v_{1}(\cdot \cap \mathbb{R} \backslash(-\varepsilon, \varepsilon)), v_{2}^{\varepsilon}=v_{2}(\cdot \cap$ $\mathbb{R} \backslash(-\varepsilon, \varepsilon))$, and $\lambda_{1}^{\varepsilon}=v_{1}^{\varepsilon}(\mathbb{R}), \lambda_{2}^{\varepsilon}=v_{2}^{\varepsilon}(\mathbb{R})$. We denote by $L_{1}^{\varepsilon}$ and $L_{2}^{\varepsilon}$ the Lévy processes corresponding to the Lévy measures $v_{1}^{\varepsilon}$ and $v_{2}^{\varepsilon}$, respectively. As before, $\mathbb{Q}_{\varepsilon}$ be a probability measure such that

$$
\left.\frac{d \mathbb{P}}{d \mathbb{Q}_{\varepsilon}}\right|_{\mathcal{F}_{t}}=V^{\varepsilon}(t), \quad t \geq 0
$$

where $X^{\varepsilon}$ solves

$$
\left\{\begin{array}{l}
d X^{\varepsilon}(t)=b\left(X^{\varepsilon}(t)\right) d t+d L_{0}(t)+d L_{1}^{\varepsilon}(t), \quad t>0 \\
X^{\varepsilon}(0)=X_{0}^{\varepsilon}
\end{array}\right.
$$

and $V^{\varepsilon}$ solves

$$
\left\{\begin{array}{l}
d V^{\varepsilon}(t)=V^{\varepsilon}(t) g\left(X^{\varepsilon}(t)\right)\left[d W_{2}(t)+g\left(X^{\varepsilon}(t)\right) d t\right] \\
V^{\varepsilon}(0)=1
\end{array}\right.
$$

Let $\rho^{\varepsilon}=\left\{\rho_{t}^{\varepsilon}: t \geq 0\right\}$ be the un-normalized conditional density process given by

$$
\rho_{t}^{\varepsilon}(f)=\mathbb{E}^{\mathbb{Q}_{\varepsilon}}\left[V^{\varepsilon}(t) f\left(X^{\varepsilon}(t)\right) \mid \mathcal{Y}_{t}^{\varepsilon}\right]
$$

and $Y^{\varepsilon}=\left\{Y^{\varepsilon}(t): t \geq 0\right\}$ be the solution to

$$
\left\{\begin{array}{l}
d Y^{\varepsilon}(t)=g\left(X^{\varepsilon}(t)\right) d t+d L_{2}^{\varepsilon}(t)+d W_{2}(t), \quad t>0 \\
Y^{\varepsilon}(0)=Y_{0}^{\varepsilon}
\end{array}\right.
$$

Notice that under the probability measure $\mathbb{Q}_{\varepsilon}$, the continuous part of $Y^{\varepsilon}$ is a Brownian motion.

Let us denote the number of jumps of $L_{2}^{\varepsilon}$ in the time interval $[0, t]$ by $N_{\varepsilon}(t)$, the jumps themselves by $\left\{Y_{2, \varepsilon, i}: i=1, \ldots, N_{\varepsilon}(t)\right\}$, and the jump times by $\left\{s_{i}^{\varepsilon}: i=\right.$ $\left.1, \ldots, N_{\varepsilon}(t)\right\}$. Then,

$$
L_{1}^{\varepsilon}(t)=\sum_{i=1}^{N_{\varepsilon}(t)} Y_{Y_{2, \varepsilon, i}}^{1, \varepsilon, i}, \quad t \geq 0
$$

where $\left\{Y_{Y_{2, \varepsilon, i}}^{1, \varepsilon, i}: i=1, \ldots, N_{\varepsilon}(t)\right\}$ is a family of independent random variables. For any $i=1, \ldots, N_{\varepsilon}(t)$, the random variable $Y_{Y_{2, \varepsilon, i}}^{1, \varepsilon, i}$ is distributed by $v_{1, z}^{\varepsilon} / \lambda_{1}^{\varepsilon}$ with $z=Y_{2, \varepsilon, i}$. Now following the same calculations as in the proof of Theorem 2.2, we get

$$
\begin{aligned}
f\left(X^{\varepsilon}(t)\right)= & f\left(X_{0}^{\varepsilon}\right)+\int_{0}^{t}\left(\mathcal{A}_{0} f\right)\left(X^{\varepsilon}(s)\right) d s+M_{\varepsilon}(t) \\
& +\sum_{1 \leq i \leq N_{\varepsilon}(t)} f\left(X^{\varepsilon}\left(s_{i}^{-}\right)+Y_{Y_{2, \varepsilon, i}}^{1, \varepsilon, i}\right)-f\left(X^{\varepsilon}\left(s_{i}^{-}\right)\right)
\end{aligned}
$$


where $M_{\varepsilon}$ is a martingale and $\mathbb{E}^{\mathbb{Q}}\left[M_{\varepsilon}(t) \mid \mathcal{Y}_{t}\right]=0$. Put

$$
v_{1, z_{2}}^{\varepsilon}(U)=\int_{U \cap[(-\infty,-\varepsilon] \cup[\varepsilon, \infty)]} h\left(z_{1}, z_{2}\right) v_{1}\left(d z_{1}\right), \quad U \in \mathcal{B}(\mathbb{R}) .
$$

Similarly as in Theorem 2.2, we denote the Poisson random measure corresponding to $L_{2}^{\varepsilon}$ by $\eta_{2}^{\varepsilon}$. Thus, we can write

$$
\begin{aligned}
f\left(X^{\varepsilon}(t)\right)= & f\left(X_{0}^{\varepsilon}\right)+\int_{0}^{t}\left(\mathcal{A}_{0} f\right)\left(X^{\varepsilon}(s)\right) d s+M_{\varepsilon}(t) \\
& +\sum_{1 \leq i \leq N_{\varepsilon}(t)} f\left(X^{\varepsilon}\left(s_{i}^{-}\right)+Y_{Y_{2, \varepsilon, i}, \varepsilon, i}\right)-f\left(X^{\varepsilon}\left(s_{i}^{-}\right)\right) \\
= & f\left(X_{0}^{\varepsilon}\right)+\int_{0}^{t}\left(\mathcal{A}_{0} f\right)\left(X^{\varepsilon}(s)\right) d s+M_{\varepsilon}(t) \\
& +\sum_{1 \leq i \leq N_{\varepsilon}(t)} f\left(X^{\varepsilon}\left(s_{i}^{-}\right)+Y_{Y_{2, \varepsilon, i}, \varepsilon, i}\right)-f\left(X^{\varepsilon}\left(s_{i}^{-}\right)\right) \\
& -\int_{0}^{t} \int_{\mathbb{R}} \int_{\mathbb{R}}\left[f\left(X^{\varepsilon}\left(s_{i}^{-}\right)+z_{1}\right)-f\left(X^{\varepsilon}\left(s_{i}^{-}\right)\right)\right] v_{1, z_{2}}^{\varepsilon}\left(d z_{1}\right) \eta_{2}^{\varepsilon}\left(d z_{2}, d s\right) \\
& +\int_{0}^{t} \int_{\mathbb{R}} \int_{\mathbb{R}}\left[f\left(X^{\varepsilon}\left(s_{i}^{-}\right)+z_{1}\right)-f\left(X^{\varepsilon}\left(s_{i}^{-}\right)\right)\right] v_{1, z_{2}}^{\varepsilon}\left(d z_{1}\right) \eta_{2}^{\varepsilon}\left(d z_{2}, d s\right) \\
= & f\left(X_{0}^{\varepsilon}\right)+\int_{0}^{t} \mathcal{A}_{0} f\left(X^{\varepsilon}(s)\right) d s+M_{\varepsilon}(t)+\tilde{M}_{\varepsilon}(t) \\
& +\int_{0}^{t} \int_{\mathbb{R}} \int_{\mathbb{R}}\left[f\left(X^{\varepsilon}\left(s_{i}^{-}\right)+z_{1}\right)-f\left(X^{\varepsilon}\left(s_{i}^{-}\right)\right)\right] v_{1, z_{2}}^{\varepsilon}\left(d z_{1}\right) \eta_{2}^{\varepsilon}\left(d z_{2}, d s\right) .
\end{aligned}
$$

By using same arguments in the proof of Theorem (2.2), we can show that for $t \geq 0$ we have $\mathbb{E}^{\mathbb{Q}_{\varepsilon}}\left[\tilde{M}_{\varepsilon}(t) \mid \mathcal{Y}_{t}^{\varepsilon}\right]=0$. Next, the process $V^{\varepsilon}=\left\{V^{\varepsilon}(t): t \geq 0\right\}$ satisfies under $\mathbb{Q}_{\varepsilon}$ the stochastic differential equation

$$
d V^{\varepsilon}(t)=V^{\varepsilon}(t) g\left(X^{\varepsilon}(t)\right) d Y^{c}(t), \quad t>0, \quad V^{\varepsilon}(0)=1,
$$

where $Y^{c}$ denotes the continuous part of $Y^{\varepsilon}$ and it does not depend up on $\varepsilon$. Since $V^{\varepsilon}$ is driven by the continuous part of $Y^{\varepsilon}$ and the jumps times are given, there will be no correlation terms in the formula for $V^{\varepsilon}(t)$. Thus, we get

$$
\begin{aligned}
f\left(X^{\varepsilon}(t)\right) V^{\varepsilon}(t)= & f\left(X_{0}^{\varepsilon}\right)+\int_{0}^{t} V^{\varepsilon}\left(s^{-}\right) d M_{\varepsilon}(s)+\int_{0}^{t} V^{\varepsilon}\left(s^{-}\right) d \tilde{M}_{\varepsilon}(s) \\
& +\int_{0}^{t} \int_{\mathbb{R}} \int_{\mathbb{R}} V^{\varepsilon}\left(s^{-}\right)\left[f\left(X^{\varepsilon}\left(s^{-}\right)+z_{1}\right)-f\left(X^{\varepsilon}\left(s^{-}\right)\right)\right] \\
& \times v_{1, z_{2}}^{\varepsilon}\left(d z_{1}\right) \eta_{2}^{\varepsilon}\left(d z_{2}, d s\right) \\
& +\int_{0}^{t} V^{\varepsilon}(s) f\left(X^{\varepsilon}(s)\right) d Y^{c}(s)+\int_{0}^{t} V^{\varepsilon}(s)\left(\mathcal{A}_{0} f\right)\left(X^{\varepsilon}(s)\right) d s .
\end{aligned}
$$


Note that

$$
\mathcal{Y}_{t}^{\varepsilon}=\sigma\left\{Y_{r}: 0 \leq r \leq t, \varepsilon \leq\left|\Delta L_{2}(r)\right|<\infty\right\} .
$$

Taking into account that $M_{\varepsilon}$ is a martingale over $\left(\Omega, \mathcal{F},\left(\mathcal{Y}_{t}^{\varepsilon}\right)_{t \geq 0}, \mathbb{Q} \varepsilon\right)$, the fact that $\mathbb{E}^{\mathbb{Q}_{\varepsilon}}\left[\tilde{M}_{\varepsilon}(t) \mid \mathcal{Y}_{t}^{\varepsilon}\right]=0$ and taking the conditional expectation together with the Fubini theorem (Applebaum (2009), Theorem 1.1.8), we get

$$
\begin{aligned}
\mathbb{E}^{\mathbb{Q}_{\varepsilon}}\left[f\left(X^{\varepsilon}(t)\right) V^{\varepsilon}(t) \mid \mathcal{Y}_{t}^{\varepsilon}\right] \\
=\mathbb{E}^{\mathbb{Q}_{\varepsilon}\left[f\left(X_{0}^{\varepsilon}\right) \mid \mathcal{Y}_{0}\right]} \\
+\underbrace{\mathbb{E}^{\mathbb{Q}_{\varepsilon}}\left[\int_{0}^{t} V^{\varepsilon}\left(s^{-}\right) d M_{\varepsilon}(s) \mid \mathcal{Y}_{t}^{\varepsilon}\right]}_{=0}+\underbrace{\mathbb{E}^{\mathbb{Q}_{\varepsilon}}\left[\int_{0}^{t} V^{\varepsilon}\left(s^{-}\right) d \tilde{M}_{\varepsilon}(s) \mid \mathcal{Y}_{t}^{\varepsilon}\right]}_{=0} \\
+\mathbb{E}^{\mathbb{Q}_{\varepsilon}}\left[\int_{0}^{t} V^{\varepsilon}\left(s^{-}\right) f\left(X^{\varepsilon}\left(s^{-}\right)\right) d Y^{c, \varepsilon}(s) \mid \mathcal{Y}_{t}^{\varepsilon}\right] \\
+\mathbb{E}^{\mathbb{Q}_{\varepsilon}}\left[\int_{0}^{t} V^{\varepsilon}\left(s^{-}\right)\left(\mathcal{A}_{0} f\right)\left(X^{\varepsilon}\left(s^{-}\right)\right) d s \mid \mathcal{Y}_{t}^{\varepsilon}\right] \\
+\mathbb{E}^{\mathbb{Q}_{\varepsilon}}\left[\int_{0}^{t} \int_{\mathbb{R}} \int_{\mathbb{R}} V^{\varepsilon}\left(s^{-}\right)\left[f\left(X^{\varepsilon}\left(s^{-}\right)+z_{1}\right)-f\left(X^{\varepsilon}\left(s^{-}\right)\right)\right]\right. \\
\left.\quad \times v_{1, z_{2}}^{\varepsilon}\left(d z_{1}\right) \eta_{2}^{\varepsilon}\left(d z_{2}, d s\right) \mid \mathcal{Y}_{t}^{\varepsilon}\right] .
\end{aligned}
$$

By imitating the calculation (2.13) and (2.14) for

$$
\int_{0}^{t} V^{\varepsilon}\left(s^{-}\right) d \tilde{M}_{\varepsilon}(s)=\int_{0}^{t} V^{\varepsilon}\left(s^{-}\right) d J^{\varepsilon}(s)-\int_{0}^{t} V^{\varepsilon}\left(s^{-}\right) d R^{\varepsilon}(s),
$$

we can again show that

$$
\mathrm{E}^{\mathbb{Q}_{\varepsilon}}\left[\int_{0}^{t} V^{\varepsilon}\left(s^{-}\right) d \tilde{M}_{\varepsilon}(s) \mid \mathcal{Y}_{t}^{\varepsilon}\right]=0 .
$$

Next by following the same calculations done in (2.15) and (2.16), we can prove that

$$
\mathrm{E}^{\mathbb{Q}_{\varepsilon}}\left[\int_{0}^{t} V^{\varepsilon}\left(s^{-}\right) d M_{\varepsilon}(s) \mid \mathcal{Y}_{t}^{\varepsilon}\right]=0 .
$$

Now, since $X^{\varepsilon}(s)$ is $\mathcal{F}_{S}$-measurable we have

$$
\mathbb{E}^{\mathbb{Q}_{\varepsilon}}\left[V^{\varepsilon}(s)\left(\mathcal{A}_{0} f\right)\left(X^{\varepsilon}(s)\right) \mid \mathcal{Y}_{t}^{\varepsilon}\right]=\mathbb{E}^{\mathbb{Q}_{\varepsilon}}\left[V^{\varepsilon}(s)\left(\mathcal{A}_{0} f\right)\left(X^{\varepsilon}(s)\right) \mid \mathcal{Y}_{s}^{\varepsilon}\right] .
$$

Note that since $Y^{c}(t)$ is $\mathcal{Y}_{t}^{\varepsilon}$-measurable, we have similarly as in Theorem 2.2,

$$
\begin{aligned}
\mathbb{E}^{\mathbb{Q}_{\varepsilon}}[ & \left.\int_{0}^{t} V^{\varepsilon}\left(s^{-}\right) g\left(X^{\varepsilon}\left(s^{-}\right)\right) f\left(X^{\varepsilon}\left(s^{-}\right)\right) d Y_{s}^{c} \mid \mathcal{Y}_{t}^{\varepsilon}\right] \\
& =\int_{0}^{t} \mathbb{E}^{\mathbb{Q}_{\varepsilon}}\left[V^{\varepsilon}\left(s^{-}\right) g\left(X^{\varepsilon}\left(s^{-}\right)\right) f\left(X^{\varepsilon}\left(s^{-}\right)\right) \mid \mathcal{Y}_{s^{-}}^{\varepsilon}\right] d Y_{s}^{c} .
\end{aligned}
$$


Since $V^{\varepsilon}\left(s^{-}\right) \int_{\mathbb{R}}\left[f\left(X^{\varepsilon}\left(s_{i}^{-}\right)+y\right)-f\left(X^{\varepsilon}\left(s_{i}^{-}\right)\right)\right] v_{1, z_{2}}^{\varepsilon}(d y)$ is an $\mathcal{F}_{s^{-}}$-measurable random variable, $L_{2}$ is a Lévy process with respect to $\left(\Omega, \mathcal{Y},\left(\mathcal{Y}_{t}^{\varepsilon}\right)_{t \geq 0}, \mathbb{Q}^{\varepsilon}\right)$, we obtain

$$
\begin{aligned}
\mathbb{E}^{\mathbb{Q}_{\varepsilon}}\left[\int_{0}^{t} \int_{\mathbb{R}} \int_{\mathbb{R}} V^{\varepsilon}\left(s^{-}\right)\left[f\left(X^{\varepsilon}\left(s^{-}\right)+z_{1}\right)-f\left(X^{\varepsilon}\left(s^{-}\right)\right)\right] v_{1, z_{2}}^{\varepsilon}\left(d z_{1}\right) \eta_{2}^{\varepsilon}\left(d z_{2}, d s\right) \mid \mathcal{Y}_{t}^{\varepsilon}\right] \\
=\int_{0}^{t} \int_{\mathbb{R}} \mathbb{E}^{\mathbb{Q}_{\varepsilon}}\left[\int_{\mathbb{R}} V^{\varepsilon}\left(s^{-}\right)\left[f\left(X^{\varepsilon}\left(s^{-}\right)+z_{1}\right)-f\left(X^{\varepsilon}\left(s^{-}\right)\right)\right] v_{1, z_{2}}^{\varepsilon}\left(d z_{1}\right) \mid \mathcal{Y}_{t}^{\varepsilon}\right] \\
\quad \times \eta_{2}^{\varepsilon}\left(d z_{2}, d s\right) \\
=\int_{0}^{t} \int_{\mathbb{R}} \int_{\mathbb{R}} \mathbb{E}^{\mathbb{Q}_{\varepsilon}}\left[V^{\varepsilon}\left(s^{-}\right)\left[f\left(X^{\varepsilon}\left(s^{-}\right)+z_{1}\right)-f\left(X^{\varepsilon}\left(s^{-}\right)\right)\right] \mid \mathcal{Y}_{s^{-}}^{\varepsilon}\right] \\
\quad \times v_{1, z_{2}}^{\varepsilon}\left(d z_{1}\right) \eta_{2}^{\varepsilon}\left(d z_{2}, d s\right) .
\end{aligned}
$$

Now collecting all the terms, we get

$$
\begin{aligned}
& \mathbb{E}^{\mathbb{Q}_{\varepsilon}}\left[f\left(X^{\varepsilon}(t)\right) V^{\varepsilon}(t) \mid \mathcal{Y}_{t}^{\varepsilon}\right] \\
&=\mathbb{E}^{\mathbb{Q}_{\varepsilon}}\left[f\left(X_{0}^{\varepsilon}\right) \mid \mathcal{Y}_{0}\right]+\int_{0}^{t} \mathbb{E}^{\mathbb{Q}_{\varepsilon}}\left[V^{\varepsilon}(s)\left(\mathcal{A}_{0} f\right)\left(X^{\varepsilon}(s)\right) \mid \mathcal{Y}_{s}^{\varepsilon}\right] d s \\
&+\int_{0}^{t} \mathbb{E}^{\mathbb{Q}_{\varepsilon}}\left[g\left(X^{\varepsilon}(s)\right) f\left(X^{\varepsilon}(s)\right) \mid \mathcal{Y}_{s}^{\varepsilon}\right] d Y^{c}(s) \\
&+\int_{0}^{t} \int_{\mathbb{R}} \int_{\mathbb{R}} \mathbb{E}^{\mathbb{Q}_{\varepsilon}}\left[V^{\varepsilon}\left(s^{-}\right)\left[f\left(X^{\varepsilon}\left(s^{-}\right)+z_{1}\right)-f\left(X^{\varepsilon}\left(s^{-}\right)\right)\right] \mid \mathcal{Y}_{s^{-}}^{\varepsilon}\right] \\
& \times v_{1, z_{2}}^{\varepsilon}\left(d z_{1}\right) \eta_{2}^{\varepsilon}\left(d z_{2}, d s\right) .
\end{aligned}
$$

Now we would like to pass to the limit and to get the desired Zakai equation. By Applebaum (2009), page 235, Corollary 4.3.10 and page 392, Theorem 6.5.2, it follows $X^{\varepsilon} \rightarrow X$ and $Y^{\varepsilon} \rightarrow Y$ uniformly on compact interval almost surely. Hence, the term $\mathbb{E}^{\mathbb{Q}_{\varepsilon}}\left[f\left(X_{0}^{\varepsilon}\right) \mid \mathcal{Y}_{0}\right]$ converges to $\mathbb{E}^{\mathbb{Q}}\left[f\left(X_{0}\right) \mid \mathcal{Y}_{0}\right]$ as $\varepsilon \rightarrow 0$. Fix $t \geq 0$. Because of the above fact, we apply Theorem C.1 to show that for any $s \in[0, t], \mathbb{Q}$-a.s.

$$
\mathbb{E}^{\mathbb{Q}_{\varepsilon}}\left[V^{\varepsilon}(s)\left(\mathcal{A}_{0} f\right)\left(X^{\varepsilon}(s)\right) \mid \mathcal{Y}_{s}^{\varepsilon}\right] \rightarrow \mathbb{E}^{\mathbb{Q}}\left[V(s)\left(\mathcal{A}_{0} f\right)(X(s)) \mid \mathcal{Y}_{s}\right], \quad \varepsilon \rightarrow 0 .
$$

The Lebesgue dominated convergence theorem gives that

$$
\int_{0}^{t} \mathbb{E}^{\mathbb{Q}_{\varepsilon}}\left[V^{\varepsilon}(s)\left(\mathcal{A}_{0} f\right)\left(X^{\varepsilon}(s)\right) \mid \mathcal{Y}_{s}^{\varepsilon}\right] d s
$$

converges to $\int_{0}^{t} \mathbb{E}^{\mathbb{Q}}\left[V(s)\left(\mathcal{A}_{0} f\right)(X(s)) \mid \mathcal{Y}_{s}\right] d s$. Next, again applying Theorem C. 1 gives for any $s \in[0, t]$ that $\mathbb{E}^{\mathbb{Q}_{\varepsilon}}\left[g\left(X^{\varepsilon}(s)\right) f\left(X^{\varepsilon}(s)\right) \mid \mathcal{Y}_{s}^{\varepsilon}\right]$ converges to $\mathbb{E}^{\mathbb{Q}}\left[g(X(s)) f(X(s)) \mid \mathcal{Y}_{s}\right]$. Again the Burkholder-Gundy-Davis inequality and the Lebesgue dominated convergence theorem gives that

$$
\int_{0}^{t} \mathbb{E}^{\mathbb{Q}_{\varepsilon}}\left[g\left(X^{\varepsilon}(s)\right) f\left(X^{\varepsilon}(s)\right) \mid \mathcal{Y}_{s}^{\varepsilon}\right] d Y^{c}(s)
$$


converges to

$$
\int_{0}^{t} \mathbb{E}^{\mathbb{Q}}\left[g(X(s)) f(X(s)) \mid \mathcal{Y}_{s}\right] d Y^{c}(s)
$$

as $\varepsilon \rightarrow 0$. Our final goal is to prove that

$$
\begin{aligned}
& \int_{0}^{t} \int_{\mathbb{R}} \int_{\mathbb{R}} \mathbb{E}^{\mathbb{Q}_{\varepsilon}}\left[V^{\varepsilon}\left(s^{-}\right)\left[f\left(X^{\varepsilon}\left(s^{-}\right)+z_{1}\right)-f\left(X^{\varepsilon}\left(s^{-}\right)\right)\right] \mid \mathcal{Y}_{s^{-}}^{\varepsilon}\right] \\
& \quad \times v_{1, z_{2}}^{\varepsilon}\left(d z_{1}\right) \eta_{2}^{\varepsilon}\left(d z_{2}, d s\right)
\end{aligned}
$$

converges to

$$
\int_{0}^{t} \int_{\mathbb{R}} \int_{\mathbb{R}} \mathbb{E}^{\mathbb{Q}}\left[V\left(s^{-}\right)\left[f\left(X\left(s^{-}\right)+z_{1}\right)-f\left(X\left(s^{-}\right)\right)\right] \mid \mathcal{Y}_{s^{-}}\right] v_{1, z_{2}}\left(d z_{1}\right) \eta_{2}\left(d z_{2}, d s\right)
$$

as $\varepsilon \rightarrow 0$. For the notational convenient, we use

$$
\mathscr{U}_{t, z_{1}, z_{2}}^{\varepsilon}=\mathbb{E}^{\mathbb{Q}_{\varepsilon}}\left[V^{\varepsilon}\left(t^{-}\right)\left[f\left(X^{\varepsilon}\left(t^{-}\right)+z_{1}\right)-f\left(X^{\varepsilon}\left(t^{-}\right)\right)\right] \mid \mathcal{Y}_{t^{-}}^{\varepsilon}\right]
$$

and

$$
\mathscr{U}_{t, z_{1}, z_{2}}=\mathbb{E}^{\mathbb{Q}}\left[V\left(t^{-}\right)\left[f\left(X\left(t^{-}\right)+z_{1}\right)-f\left(X\left(t^{-}\right)\right)\right] \mid \mathcal{Y}_{t^{-}}\right] .
$$

Now consider

$$
\begin{aligned}
\mathbb{E}^{\mathbb{Q}} \mid \int_{0}^{t} & \int_{\mathbb{R}} \int_{\mathbb{R}}\left[\mathscr{U}_{s, z_{1}, z_{2}}^{\varepsilon} v_{1, z_{2}}^{\varepsilon}\left(d z_{1}\right) \eta_{2}^{\varepsilon}\left(d z_{2}, d s\right)\right. \\
& \left.-\mathscr{U}_{s, z_{1}, z_{2}} v_{1, z_{2}}\left(d z_{1}\right) \eta_{2}\left(d z_{2}, d s\right)\right] \mid \\
\leq & \mathbb{E}^{\mathbb{Q}} \mid \int_{0}^{t} \int_{\mathbb{R}} \int_{\mathbb{R}}\left[\mathscr{U}_{s, z_{1}, z_{2}}^{\varepsilon} \mathbf{1}_{(-\varepsilon, \varepsilon)^{c}}\left(z_{1}\right)-\mathscr{U}_{s, z_{1}, z_{2}}\right] \\
& \times h\left(z_{1}, z_{2}\right) \nu_{1}\left(d z_{1}\right) \eta_{2}^{\varepsilon}\left(d z_{2}, d s\right) \mid \\
& +\mathbb{E}^{\mathbb{Q}} \mid \int_{0}^{t} \int_{\mathbb{R}} \int_{\mathbb{R}} \mathscr{U}_{s, z_{1}, z_{2}} h\left(z_{1}, z_{2}\right) \nu_{1}\left(d z_{1}\right) \\
& \times\left[\eta_{2}^{\varepsilon}\left(d z_{2}, d s\right)-\eta_{2}\left(d z_{2}, d s\right)\right] \mid
\end{aligned}
$$

The first term in right-hand side gives

$$
\begin{aligned}
\mathbb{E}^{\mathbb{Q}} \mid \int_{0}^{t} \int_{\mathbb{R}} \int_{\mathbb{R}}\left[\mathscr{U}_{s, z_{1}, z_{2}}^{\varepsilon} \mathbf{1}_{(-\varepsilon, \varepsilon)^{c}}\left(z_{1}\right)-\mathscr{U}_{s, z_{1}, z_{2}}\right] \\
\quad \times h\left(z_{1}, z_{2}\right) \nu_{1}\left(d z_{1}\right) \eta_{2}^{\varepsilon}\left(d z_{2}, d s\right) \mid \\
\leq \mathbb{E}^{\mathbb{Q}} \mid \int_{0}^{t} \int_{\mathbb{R}} \int_{\mathbb{R}}\left[\mathscr{U}_{s, z_{1}, z_{2}}^{\varepsilon} \mathbf{1}_{(-\varepsilon, \varepsilon)^{c}}\left(z_{1}\right)-\mathscr{U}_{s, z_{1}, z_{2}}\right]
\end{aligned}
$$




$$
\begin{aligned}
& \times h\left(z_{1}, z_{2}\right) v_{1}\left(d z_{1}\right) \tilde{\eta}_{2}^{\varepsilon}\left(d z_{2}, d s\right) \mid \\
& +\mathbb{E}^{\mathbb{Q}} \mid \int_{0}^{t} \int_{\mathbb{R}} \int_{\mathbb{R}}\left[\mathscr{U}_{s, z_{1}, z_{2}}^{\varepsilon} \mathbf{1}_{(-\varepsilon, \varepsilon)^{c}}\left(z_{1}\right)-\mathscr{U}_{s, z_{1}, z_{2}}\right] \\
& \times h\left(z_{1}, z_{2}\right) v_{1}\left(d z_{1}\right) \mathbf{1}_{(-\varepsilon, \varepsilon)^{c}}\left(z_{2}\right) v_{2}\left(d z_{2}\right) d s \mid,
\end{aligned}
$$

where $\tilde{\eta}_{2}^{\varepsilon}\left(d z_{2}, d s\right)=\eta_{2}^{\varepsilon}\left(d z_{2}, d s\right)-v_{2}^{\varepsilon}\left(d z_{2}\right) d s$ and $(-\varepsilon, \varepsilon)^{c}=\mathbb{R} \backslash(-\varepsilon, \varepsilon)$. Applying the Burkholder-Gundy-Davis inequality, Hölder inequality and Jensen's inequality

$$
\begin{aligned}
& \cdots \leq C(t) \mathbb{E}^{\mathbb{Q}} \mid \int_{0}^{t} \int_{\mathbb{R}}\left[\int_{\mathbb{R}}\left(\mathscr{U}_{s, z_{1}, z_{2}}^{\varepsilon} \mathbf{1}_{(-\varepsilon, \varepsilon)^{c}}\left(z_{1}\right)-\mathscr{U}_{s, z_{1}, z_{2}}\right) h\left(z_{1}, z_{2}\right) v_{1}\left(d z_{1}\right)\right]^{2} \\
& \times\left.\mathbf{1}_{(-\varepsilon, \varepsilon)^{c}}\left(z_{2}\right) v_{2}\left(d z_{2}\right) d s\right|^{\frac{1}{2}} \\
& +\mathbb{E}^{\mathbb{Q}} \mid \int_{0}^{t} \int_{\mathbb{R}} \int_{\mathbb{R}}\left[\mathscr{U}_{s, z_{1}, z_{2}}^{\varepsilon} \mathbf{1}_{(-\varepsilon, \varepsilon)^{c}}\left(z_{1}\right)-\mathscr{U}_{s, z_{1}, z_{2}}\right] h\left(z_{1}, z_{2}\right) v_{1}\left(d z_{1}\right) \\
& \times \mathbf{1}_{(-\varepsilon, \varepsilon)^{c}}\left(z_{2}\right) v_{2}\left(d z_{2}\right) d s \\
& \leq C(t)\left(\int_{0}^{t} \int_{\mathbb{R}} \mathbb{E}^{\mathbb{Q}}\left|\int_{\mathbb{R}}\left(\mathscr{U}_{s, z_{1}, z_{2}}^{\varepsilon} \mathbf{1}_{(-\varepsilon, \varepsilon)^{c}}\left(z_{1}\right)-\mathscr{U}_{s, z_{1}, z_{2}}\right) h\left(z_{1}, z_{2}\right) v_{1}\left(d z_{1}\right)\right|^{2}\right. \\
& \left.\times \mathbf{1}_{(-\varepsilon, \varepsilon)^{c}}\left(z_{2}\right) v_{2}\left(d z_{2}\right) d s\right)^{\frac{1}{2}} \\
& +\int_{0}^{t} \int_{\mathbb{R}} \mathbb{E}^{\mathbb{Q}}\left|\int_{\mathbb{R}}\left[\mathscr{U}_{s, z_{1}, z_{2}}^{\varepsilon} \mathbf{1}_{(-\varepsilon, \varepsilon)^{c}}\left(z_{1}\right)-\mathscr{U}_{s, z_{1}, z_{2}}\right] h\left(z_{1}, z_{2}\right) v_{1}\left(d z_{1}\right)\right| \\
& \times \mathbf{1}_{(-\varepsilon, \varepsilon)^{c}}\left(z_{2}\right) v_{2}\left(d z_{2}\right) d s .
\end{aligned}
$$

Due to Assumption 2.19 and using simple arguments together with Theorem C.1 and Lebesgue Dominated Convergence theorem, we can show that the two terms in above inequality, that is,

$$
\mathbb{E}^{\mathbb{Q}}\left|\int_{\mathbb{R}}\left[\mathscr{U}_{s, z_{1}, z_{2}}^{\varepsilon} \mathbf{1}_{(-\varepsilon, \varepsilon)^{c}}\left(z_{1}\right)-\mathscr{U}_{s, z_{1}, z_{2}}\right] h\left(z_{1}, z_{2}\right) \nu_{1}\left(d z_{1}\right)\right|^{2}
$$

and

$$
\mathbb{E}^{\mathbb{Q}}\left|\int_{\mathbb{R}}\left[\mathscr{U}_{s, z_{1}, z_{2}}^{\varepsilon} \mathbf{1}_{(-\varepsilon, \varepsilon)^{c}}\left(z_{1}\right)-\mathscr{U}_{s, z_{1}, z_{2}}\right] h\left(z_{1}, z_{2}\right) v_{1}\left(d z_{1}\right)\right|
$$

converge to zero as $\varepsilon \rightarrow 0$. Then by the Lebesgue Dominated Convergence theorem, the two terms in right-hand side of above inequality converge to zero as 
$\varepsilon \rightarrow 0$. Let us consider the second term in the right-hand side of (2.25),

$$
\begin{aligned}
\mathbb{E}^{\mathbb{Q}} \mid \int_{0}^{t} & \int_{\mathbb{R}} \int_{\mathbb{R}} \mathscr{U}_{s, z_{1}, z_{2}} h\left(z_{1}, z_{2}\right) v_{1}\left(d z_{1}\right)\left[\eta_{2}^{\varepsilon}\left(d z_{2}, d s\right)-\eta_{2}\left(d z_{2}, d s\right)\right] \mid \\
\leq & \mathbb{E}^{\mathbb{Q}} \mid \int_{0}^{t} \int_{\mathbb{R}} \int_{\mathbb{R}} \mathscr{U}_{s, z_{1}, z_{2}} h\left(z_{1}, z_{2}\right) v_{1}\left(d z_{1}\right) \\
& \times\left[\tilde{\eta}_{2}^{\varepsilon}\left(d z_{2}, d s\right)-\tilde{\eta}_{2}\left(d z_{2}, d s\right)\right] \mid \\
& +\mathbb{E}^{\mathbb{Q}}\left|\int_{0}^{t} \int_{\mathbb{R}} \int_{\mathbb{R}} \mathscr{U}_{s, z_{1}, z_{2}} h\left(z_{1}, z_{2}\right) v_{1}\left(d z_{1}\right) \mathbf{1}_{(-\varepsilon, \varepsilon)}\left(z_{2}\right) v_{2}\left(d z_{2}\right) d s\right|
\end{aligned}
$$

The Burkholder-Gundy-Davis inequality and Jensen's inequality imply

$$
\begin{aligned}
\cdots \leq & \left.\left.\mathbb{E}^{\mathbb{Q}}\left|\int_{0}^{t} \int_{\mathbb{R}}\right| \int_{\mathbb{R}} \mathscr{U}_{s, z_{1}, z_{2}} h\left(z_{1}, z_{2}\right) v_{1}\left(d z_{1}\right)\right|^{2} \mathbf{1}_{(-\varepsilon, \varepsilon)}\left(z_{2}\right) v_{2}\left(d z_{2}\right) d s\right|^{\frac{1}{2}} \\
& +\mathbb{E}^{\mathbb{Q}}\left|\int_{0}^{t} \int_{\mathbb{R}} \int_{\mathbb{R}} \mathscr{U}_{s, z_{1}, z_{2}} h\left(z_{1}, z_{2}\right) v_{1}\left(d z_{1}\right) \mathbf{1}_{(-\varepsilon, \varepsilon)}\left(z_{2}\right) v_{2}\left(d z_{2}\right) d s\right| \\
\leq & {\left[\int_{0}^{t} \mathbb{E}^{\mathbb{Q}}\left|\int_{\mathbb{R}} \int_{\mathbb{R}} \mathscr{U}_{s, z_{1}, z_{2}} v_{1, z_{2}}\left(d z_{1}\right)\right|^{2} \mathbf{1}_{(-\varepsilon, \varepsilon)}\left(z_{2}\right) \nu_{2}\left(d z_{2}\right) d s\right]^{\frac{1}{2}} } \\
& +\int_{0}^{t} \int_{\mathbb{R}} \mathbb{E}^{\mathbb{Q}}\left|\int_{\mathbb{R}} \mathscr{U}_{s, z_{1}, z_{2}} v_{1, z_{2}}\left(d z_{1}\right)\right| \mathbf{1}_{(-\varepsilon, \varepsilon)}\left(z_{2}\right) v_{2}\left(d z_{2}\right) d s .
\end{aligned}
$$

Again, arguing as before and using assumption 2.19, we see that the two terms in right-hand side of the above inequality go to zero as $\varepsilon \rightarrow 0$.

Summarizing, we have shown that for any $t \geq 0, \mathbb{E}^{\mathbb{Q}_{\varepsilon}}\left[f\left(X^{\varepsilon}(t)\right) V^{\varepsilon}(t) \mid \mathcal{Y}_{t}^{\varepsilon}\right]$ converges to $\mathbb{E}^{\mathbb{Q}}\left[f(X(t)) V(t) \mid \mathcal{Y}_{t}\right] \mathbb{Q}$-a.s. It is straightforward to see that the family of processes $[0, T] \ni t \mapsto \mathbb{E}^{\mathbb{Q}_{\varepsilon}}\left[f\left(X^{\varepsilon}(t)\right) V^{\varepsilon}(t) \mid \mathcal{Y}_{t}^{\varepsilon}\right]$ is tight. Hence, we know by Theorem 7.8 by Ethier and Kurtz (1986), that the process $\mathbb{E}^{\mathbb{Q}_{\varepsilon}}\left[f\left(X^{\varepsilon}(\cdot)\right) V^{\varepsilon}(\cdot) \mid \mathcal{Y}^{\varepsilon}\right]$ converges to the process $\mathbb{E}^{\mathbb{Q}}[f(X(\cdot)) V(\cdot) \mid \mathcal{Y}$. $]$ in $\mathbb{D}([0, T] ; \mathbb{R})$.

\section{Sufficient conditions for solvability of the Zakai equation}

In practice, one is often interested in entities like

$$
\mathbb{P}(X(t) \geq a), \quad a \in \mathbb{R},
$$

where $a$ is a given threshold. This correspond to the case where $f=1_{[a, \infty)}$. Unfortunately, in this case $f \notin C^{(2)}(\mathbb{R})$ and we cannot expect that equation (2.20) is well-posed. One method to handle this problem is to treat equation (2.20) by the semigroup approach. Let us denote the infinitesimal generator of the process $L_{0}$ with the drift (that is $\int_{0}^{\cdot} b(X(s)) d s$ ) by $\mathcal{A}_{0}$. If $\mathcal{A}_{0}$ generates an analytic semigroup with good smoothing property, then one can show the existence of a measure valued solution to (2.20) even for the case where $f=1_{[a, \infty)}$. If the driving process 
$L_{0}$ of the state process $X$ is a Brownian motion, then the operator $\mathcal{A}_{0}$ in the Zakai equation (2.20) is the Laplace operator with first order operator. However, if $L_{0}$ is a Lévy process of pure jump type, ${ }^{1}$ then $\mathcal{A}_{0}$ will be a pseudo differential operator.

There exists several approaches to deal with pseudo-operators arising from Lévy processes. One way is to define the operator $\mathcal{A}_{0}$ associated with the symbol $\phi_{\mathcal{A}_{0}}$ is given by ${ }^{2}$

$$
\phi_{\mathcal{A}_{0}}(\xi):=i b(x) \xi+\int_{\mathbb{R}}\left(e^{i \xi z}-1\right) \nu_{0}(d z), \quad \xi \in \mathbb{R} .
$$

Here, $L_{0}$ is a Lévy process of pure jump type with intensity $v_{0}$. For a short account on the associated symbol to a Lévy process we refer to Fernando Pani, Hausenblas and Razafimandimby (2017). More details can be found in the article of Hoh (1998), and in the books of Jacob (2000, 2002, 2005).

It can be shown that $\mathcal{A}_{0}$ with domain $D\left(\mathcal{A}_{0}\right)$ generates a strongly continuous semigroup $T_{\mathcal{A}_{0}}=\left(T_{\mathcal{A}_{0}}(t)\right)_{t \geq 0}$ on $L^{2}\left(\mathbb{R}^{d}\right)$. This semigroup can be extended (or restricted) to a semigroup acting on $H_{2}^{s}\left(\mathbb{R}^{d}\right), s \in \mathbb{R}$. By analyzing the symbol $\phi_{\mathcal{A}_{0}}$, one gets information about the smoothing properties of the semigroup $T_{\mathcal{A}_{0}}=$ $\left(T_{\mathcal{A}_{0}}(t)\right)_{t \geq 0}$.

Definition 3.1 (Compare Fernando Pani, Hausenblas and Razafimandimby (2017)). Let $L$ be a Lévy process with symbol $\psi$ and $\psi \in C^{k}\left(\mathbb{R}^{d} \backslash\{0\}\right)$ for some $k \in \mathbb{N}_{0}$. Then the Blumenthal-Getoor index of order $k$ is defined by

$$
\beta:=\inf _{\substack{\lambda>0 \\|\alpha| \leq k}}\left\{\lambda: \lim _{|\xi| \rightarrow \infty} \frac{\left|\partial_{\xi}^{\alpha} \psi(\xi)\right|}{|\xi|^{\lambda-|\alpha|}}=0\right\}
$$

Let

$$
\beta^{+}:=\inf _{\substack{\lambda>0 \\|\alpha| \leq k}}\left\{\lambda: \limsup _{|\xi| \rightarrow \infty} \frac{\left|\partial_{\xi}^{\alpha} \psi(\xi)\right|}{|\xi|^{\lambda-|\alpha|}}=0\right\}
$$

be the upper and

$$
\beta^{-}:=\inf _{\substack{\lambda>0 \\|\alpha| \leq k}}\left\{\lambda: \liminf _{|\xi| \rightarrow \infty} \frac{\left|\partial_{\xi}^{\alpha} \psi(\xi)\right|}{|\xi|^{\lambda-|\alpha|}}=0\right\},
$$

be the lower Blumenthal-Getoor index $\beta^{-}$of order $k$. Here $\alpha$ denotes a multiindex. If $k=\infty$ then Blumenthal-Getoor index of infinity order is defined by

$$
\beta:=\inf _{\substack{\lambda>0 \\ \alpha \text { is a muliindex }}}\left\{\lambda: \lim _{|\xi| \rightarrow \infty} \frac{\left|\partial_{\xi}^{\alpha} \psi(\xi)\right|}{|\xi|^{\lambda-|\alpha|}}=0\right\} .
$$

\footnotetext{
${ }^{1}$ We say that a Lévy process is of pure jump type if it has no Gaussian part.

${ }^{2}$ If $\mathcal{A}_{0}$ is the Laplacian, then $\phi_{\mathcal{A}_{0}}(\xi)=\xi^{2}$.
} 
In many cases the index can be calculated directly from the symbol and is known. A sequence of examples of the generalized Blumenthal-Getoor index, like the symmetric $\alpha$-stable process, tempered $\alpha$-stable process, Meixner process and normal inverse Gaussian process are given in Fernando Pani, Hausenblas and Razafimandimby (2017).

Depending on the lower index of $L_{0}$ and the marginal Lévy measures $v_{1}$ and $v_{2}$ of the Lévy process $L$, one can prove that there exists a unique measure valued process $\pi=\left\{\pi_{t}: t \geq 0\right\}$ such that

$$
\pi_{t}(f)=\mathbb{E}\left[f(X(t)) \mid \mathcal{Y}_{t}\right], \quad f \in B_{b}(\mathbb{R}) .
$$

Theorem 3.1. Let us assume that

- $X_{0}$ has distribution function $F$, which has a $L^{2}$-integrable density with respect to the Lebesgue measure;

- the symbol $\psi_{0}$ associated to $L_{0}$ has lower Blumenthal-Getoor index $\alpha_{0}^{-}>1$ of order two;

- $g \in H_{2}^{\delta}(\mathbb{R}) \cap C_{b}^{(2)}(\mathbb{R})$ with $\delta>1-\frac{\alpha_{0}^{-}}{2}$;

- the symbol $\phi_{\Theta_{z}}$ associated to the operator $\Theta_{z}$, has upper Blumenthal-Getoor index $\beta^{+} \leq 1$ of order two;

- there exists some function $k: \mathbb{R}_{0}^{+} \rightarrow \mathbb{R}_{0}^{+}, k(0)=0$, continuous at 0 , such that

$$
\limsup _{|\xi| \rightarrow \infty} \frac{\left|\phi_{\Theta_{z_{2}}}(\xi)\right|}{|\xi|^{\beta^{+}}} \leq k\left(z_{2}\right), \quad z_{2} \in \mathbb{R}
$$

- for simplicity, we take $L_{1}$ and $L_{2}$ with positive jumps such that

$$
\int_{\left|z_{1}\right| \leq 1}\left|z_{1}\right| v_{1}\left(d z_{1}\right)+\int_{\left|z_{2}\right| \leq 1}\left|z_{2}\right| v_{2}\left(d z_{2}\right)<\infty .
$$

In addition, if there exists a number $p \in(1,2]$ such that

$$
\frac{\beta^{+}}{\alpha_{0}^{-}}<\frac{1}{p} \text { and } \int_{\left|z_{2}\right| \leq 1}\left|k\left(z_{2}\right)\right|^{p} v_{2}\left(d z_{2}\right)<\infty,
$$

then there exists a unique normalized conditional density $\pi=\left\{\pi_{t}: t \geq 0\right\}$ such that

$$
\pi_{t}(f)=\mathbb{E}\left[f(X(t)) \mid \mathcal{Y}_{t}\right], \quad f \in B_{b}(\mathbb{R}) .
$$

Moreover for $f \in B_{b}(\mathbb{R}), \pi_{t}(f)$ is given by

$$
\pi_{t}(f)=\sigma(t) \cdot \rho_{t}(f),
$$

where $\sigma=\{\sigma(t): t \geq 0\}$ solves

$$
\sigma(t)=1+\int_{0}^{t} \rho_{s^{-}}(g) d Y_{s}^{c}, \quad t \geq 0,
$$


and $\rho=\left\{\rho_{t}: t \geq 0\right\}$ is the unique solution of the following equation

$$
\left\{\begin{array}{l}
d \rho_{t}=\mathcal{A}_{0}^{*} \rho_{t} d t+\rho_{t} g d Y_{t}^{c}+\int_{\mathbb{R}} \Theta_{z_{2}}^{*} \rho_{t}-\eta_{2}\left(d z_{2}, d t\right), \\
\rho_{0}=\pi_{0},
\end{array}\right.
$$

where $\mathcal{A}_{0}^{*}$ and $\Theta_{z_{2}}^{*}$ are adjoint operators of $\mathcal{A}_{0}$ and $\Theta_{z_{2}}$.

The adjoint operators $\mathcal{A}_{0}^{*}$ and $\Theta_{z_{2}}^{*}$ are defined as follows. Let $\sigma_{\mathcal{A}_{0}}(x, \xi)$ be the symbol of the operator $\mathcal{A}_{0}$. Therefore by using Shubin (2001), page 26, the adjoint operator representation (3.37), the symbol of the operator $\mathcal{A}_{0}^{*}$ can be read as

$$
\sigma_{\mathcal{A}_{0}^{*}}(x, \xi) \sim \sum_{|\alpha| \leq 1} \frac{\partial_{\xi}^{\alpha} D_{x}^{\alpha} \overline{\sigma_{\mathcal{A}_{0}}(x, \xi)}}{\alpha !} .
$$

Similarly, the symbol $\phi_{z}^{*}(\xi)$ of the adjoint operator $\Theta_{z_{2}}^{*}$ is given by

$$
\phi_{z}^{*}(\xi) \sim \sum_{|\alpha| \leq s} \frac{\partial_{\xi}^{\alpha} \overline{\phi_{z}(\xi)}}{\alpha !}
$$

Proof of Theorem 3.1. We apply Theorem A.1 to get an $H_{2}^{\frac{1}{2}}(\mathbb{R})$-valued solution, and then we show the existence of normalized conditional density by using the Getoor's lemma (Getoor (1975), Proposition 4.1, or Fernando and Sritharan (2013), Lemma 3.9).

In fact, if we take $\varrho=\frac{1}{2}$, then one can easily see that (3.1) and (3.2) imply that $A:=\mathcal{A}^{*}$ and $G:=\Theta^{*}$ satisfy the assumptions of Theorem A.1. By Runst and Sickel (1996), Theorem 1, page 190, we have

$$
|u g|_{H_{2}^{\varrho+\delta-\frac{1}{2}}} \leq|u|_{H_{2}^{\varrho}}|g|_{H_{2}^{\delta}}, \quad u \in H_{2}^{\varrho}(\mathbb{R}) \text { and } g \in H_{2}^{\delta}(\mathbb{R}) .
$$

Therefore, by setting $\Sigma(u)=u \cdot g$ we also see that $\Sigma$ satisfies the assumptions of Theorem A.1 as well. Hence, from these observations we see that if the assumptions of Theorem 3.1 hold, then it follows from Theorem A.1 that there exists a $H_{2}^{\frac{1}{2}}(\mathbb{R})$-valued process $\rho$, such that for any $t \geq 0$

$$
\rho_{t}(f)=\mathbb{E}^{\mathbb{Q}}\left[V(t) f(X(t)) \mid \mathcal{Y}_{t}\right], \quad f \in \mathcal{B}(\mathbb{R}) .
$$

Second, let us fix $t>0$ and set $\mathcal{G}=\mathcal{Y}_{t}$ as the $\sigma$-field on $\Omega$ and define the operator $T$ by

$$
T f(\omega)=\mathbb{E}\left[f(X(t)) \mid \mathcal{Y}_{t}\right](\omega) .
$$

It is easy to check that $T$ is a.s. linear and positive. Let $\left\{f_{n}: n \in \mathbb{N}\right\} \subset B_{b}(\mathbb{R})$ be a sequence with $0 \leq f_{n} \uparrow f$. But if $f_{n} \rightarrow f$ in $L^{\infty}(\mathbb{R})$, then one knows by Sobolev embedding theorem that $f_{n} \rightarrow f$ in $H_{2}^{-\frac{1}{2}}(\mathbb{R})$. Since for $t>0 \rho_{t}$ is $H_{2}^{\frac{1}{2}}(\mathbb{R})$-valued 
un-normalized density measure, $\rho_{t}\left(f_{n}\right) \rightarrow \rho_{t}(f)$. Here, one has to take into account that the density of $X_{0}$ belongs $\rho_{0} \in L^{2}(\mathbb{R})$. In addition, since $\rho_{t}(1)$ is well defined and invertible (see Remark 2.4), we have

$$
\pi_{t}\left(f_{n}\right)=\frac{\rho_{t}\left(f_{n}\right)}{\rho_{t}(1)} \rightarrow \frac{\rho_{t}(f)}{\rho_{t}(1)}=\pi_{t}(f) .
$$

That is $\pi_{t}\left(f_{n}\right) \rightarrow \pi_{t}(f)$. Since for $f_{n} \uparrow f, f-f_{n}$ is a.s. positive, it follows that $T\left(f-f_{n}\right)$ is also a.s. positive and, therefore, $T f_{n} \uparrow T f$.

Now, thanks to these two points we can infer from Getoor (1975), Proposition 4.1, or Fernando and Sritharan (2013), Lemma 3.9, that there exists a kernel

$$
\mu_{t}:\left(\Omega, \mathcal{Y}_{t}\right) \rightarrow(\mathbb{R}, \mathcal{B}(\mathbb{R}))
$$

such that

$$
\mathbb{E}\left[f(X(t)) \mid \mathcal{Y}_{t}\right](\omega)=\int_{\mathbb{R}} f(u) \mu_{t}(\omega, d u), \quad f \in B_{b}(\mathbb{R})
$$

In the following corollary, we present an example to illustrate the applicability of Theorem 3.1.

Corollary 3.1. Let $L_{0}$ be a tempered $\alpha$-stable Lévy process with $\alpha>1$ with Lévy measure

$$
v(U)=\int_{U}|z|^{-\alpha-1} e^{-|z|} d z, \quad U \in \mathcal{B}(\mathbb{R}),
$$

and $v_{1}, v_{2}$ are tempered $\beta$-stable subordinators, $\beta \leq 1$, with Lévy measure

$$
v(U)=\int_{U}|z|^{-\beta-1} e^{-|z|} d z, \quad U \in \mathcal{B}(\mathbb{R}) .
$$

Let $g \in H_{2}^{\delta}(\mathbb{R}) \cap C_{b}^{(2)}(\mathbb{R}) \subseteq C_{b}^{(2)}(\mathbb{R})$ with $\delta>1-\frac{\alpha_{0}^{-}}{2}$. Let $H$ be the Clayton copula with index $\theta>0$. If the distribution of $X_{0}$ has a $L^{2}$ integrable density with respect to the Lebesgue measure, then there exists a unique family of probabilities kernels $\pi=\left\{\pi_{t}: t \geq 0\right\}$ such

$$
\pi_{t}(f)=\mathbb{E}\left[f(X(t)) \mid \mathcal{Y}_{t}\right], \quad f \in B_{b}(\mathbb{R}) .
$$

Moreover for $f \in B(\mathbb{R})$ the kernel $\pi_{t}(f)$ is given by

$$
\pi_{t}(f)=\sigma(t) \cdot \rho_{t}(f),
$$

where $\sigma=\{\sigma(t): t \geq 0\}$ solves

$$
\sigma(t)=1+\int_{0}^{t} \rho_{s^{-}}(g) d Y_{s}^{c}, \quad t \geq 0
$$


and $\rho=\left\{\rho_{t}: t \geq 0\right\}$ solves

$$
\left\{\begin{array}{l}
d \rho_{t}=\mathcal{A}_{0}^{*} \rho_{t} d t+\rho_{t} h d Y_{t}^{c}+\int_{\mathbb{R}} \Theta_{z_{2}}^{*} \rho_{t}-\eta_{2}\left(d z_{2}, d t\right), \\
\rho_{0}=\pi_{0},
\end{array}\right.
$$

where

$$
\Theta_{z} f(x)=\int_{\mathbb{R}}\left[f\left(x+z_{1}\right)-f(x)\right] v_{1, z}\left(d z_{1}\right), \quad z \in \mathbb{R}_{+}, x \in \mathbb{R}, f \in C_{b}^{(2)}(\mathbb{R}) .
$$

Proof. By Theorem 1 of Runst and Sickel (1996), page 190, we have

$$
|u(r) g|_{H_{2}^{\varrho-\gamma / \alpha_{0}^{-}}}^{2} \leq|u(r)|_{H_{2}^{\varrho}}|g|_{H_{2}^{\delta}}
$$

Now fix $z \in \mathbb{R} \backslash\{0\}$. In the first step, we will investigate the symbol $\phi_{\Theta_{z}}$ of $\Theta_{z}$. The operator $\Theta_{z}$ is reduced to following the form with the Clayton copula for $f \in L^{2}(\mathbb{R})$,

$$
\begin{aligned}
\left(\Theta_{z} f\right)(x)= & (1-\theta) \int_{0}^{\infty}[f(x+y)-f(x)] \\
& \times\left(\left|U_{1}(y)\right|^{-\theta}+\left|U_{2}(z)\right|^{-\theta}\right)^{-\frac{1}{\theta}-2}\left|U_{1}(y)\right|^{-\theta-1}\left|U_{2}(z)\right|^{-\theta-1} v_{1}(d y) .
\end{aligned}
$$

For us, it is important to know the upper index of the symbol $\phi_{\Theta_{z}}$ associated to $\Theta_{z}$. The symbol $\phi_{\Theta_{z}}$ is given by

$$
\begin{aligned}
\phi_{\Theta_{z}}(\xi)= & (1+\theta) \int_{0}^{\infty}\left[e^{i \xi y}-1\right] \\
& \times\left(\left|U_{1}(y)\right|^{-\theta}+\left|U_{2}(z)\right|^{-\theta}\right)^{-\frac{1}{\theta}-2}\left|U_{1}(y)\right|^{-\theta-1}\left|U_{2}(z)\right|^{-\theta-1} v_{1}(d y) .
\end{aligned}
$$

By the Clayton copula, we get

$$
\begin{aligned}
\phi_{\Theta_{z}}(\xi)= & (1+\theta)\left|U_{2}(z)\right|^{-\theta-1} \\
& \times \int_{0}^{\infty}\left[e^{i \xi y}-1-i \xi y\right]\left(\left|U_{1}(y)\right|^{-\theta}+\left|U_{2}(z)\right|^{-\theta}\right)^{-\frac{1}{\theta}-2} \\
& \times\left|U_{1}(y)\right|^{-\theta-1} v_{1}(d y)+(1+\theta)\left|U_{2}(z)\right|^{-\theta-1} \\
& \times \int_{0}^{\infty} i \xi y\left(\left|U_{1}(y)\right|^{-\theta}+\left|U_{2}(z)\right|^{-\theta}\right)^{-\frac{1}{\theta}-2}\left|U_{1}(y)\right|^{-\theta-1} v_{1}(d y) \\
= & (1-\theta)\left|U_{2}(z)\right|^{-\theta-1} \times 2(i \xi)^{2} \int_{0}^{\infty} \int_{0}^{y} \int_{0}^{v} \\
& e^{i \xi u} d u d v\left(\left|U_{1}(y)\right|^{-\theta}+\left|U_{2}(z)\right|^{-\theta}\right)^{-\frac{1}{\theta}-2}\left|U_{1}(y)\right|^{-\theta-1} f_{1}(y) d y \\
& +(1+\theta)\left|U_{2}(z)\right|^{-\theta-1} \\
& \times \int_{0}^{\infty} i \xi y\left(\left|U_{1}(y)\right|^{-\theta}+\left|U_{2}(z)\right|^{-\theta}\right)^{-\frac{1}{\theta}-2}\left|U_{1}(y)\right|^{-\theta-1} v_{1}(d y),
\end{aligned}
$$


where $v_{1}(d y)=f_{1}(y) d y$. One gets by the Fubini's theorem

$$
\begin{aligned}
I_{1}(z)= & 2(1+\theta)\left|U_{2}(z)\right|^{-\theta-1}(i \xi)^{2} \lim _{R \rightarrow \infty} \int_{0}^{R} e^{i \xi y} \\
& \times\left[\int_{y}^{\infty} \int_{v}^{\infty}\left(\left|U_{1}(u)\right|^{-\theta}+\left|U_{2}(z)\right|^{-\theta}\right)^{-\frac{1}{\theta}-2}\left|U_{1}(u)\right|^{-\theta-1} f_{1}(u) d u d v\right] d y .
\end{aligned}
$$

Applying a version of Corput's lemma (see Stein (1993), page 334-(6)), we infer that

$$
\begin{aligned}
\left|I_{1}(z)\right| \leq & 2(1+\theta)\left|U_{2}(z)\right|^{-\theta-1}|\xi| \lim _{R \rightarrow \infty}\left|\int_{0}^{R} e^{i \xi y} d y\right| \\
& \times\left.\left|\int_{0}^{\infty} \int_{v}^{\infty}\left(\left|U_{1}(u)\right|^{-\theta}+\left|U_{2}(z)\right|^{-\theta}\right)^{-\frac{1}{\theta}-2}\right| U_{1}(u)\right|^{-\theta-1} f_{1}(u) d u d v \mid \\
\leq & 2(1+\theta)\left|U_{2}(z)\right|^{-\theta-1}|\xi| \\
& \times\left.\left|\int_{0}^{\infty} \int_{v}^{\infty}\left(\left|U_{1}(u)\right|^{-\theta}+\left|U_{2}(z)\right|^{-\theta}\right)^{-\frac{1}{\theta}-2}\right| U_{1}(u)\right|^{-\theta-1} f_{1}(u) d u d v \mid .
\end{aligned}
$$

Substitution with $m=U_{1}(u)$ gives the estimate

$$
\begin{aligned}
\left|I_{1}(z)\right| \leq & 2(1+\theta)\left|U_{2}(z)\right|^{-\theta-1} \\
& \times\left.|\xi|\left|\int_{0}^{\infty} \int_{U_{1}(v)}^{0}\left(|m|^{-\theta}+\left|U_{2}(z)\right|^{-\theta}\right)^{-\frac{1}{\theta}-2}\right| m\right|^{-\theta-1} d m d v \mid,
\end{aligned}
$$

from which we deduce that

$$
\left|I_{1}(z)\right| \leq 2(1+\theta)\left|U_{2}(z)\right|^{-\theta-1}|\xi|\left|\int_{0}^{\infty}\left(\left|U_{1}(v)\right|^{-\theta}+\left|U_{2}(z)\right|^{-\theta}\right)^{-\frac{1}{\theta}-1} d v\right| .
$$

Again, substitution with $l=U_{1}(v)$ gives

$$
\begin{aligned}
\left|I_{1}(z)\right| \leq & 2(1+\theta)\left|U_{2}(z)\right|^{-\theta-1}|\xi| \\
& \times\left|\int_{0}^{\infty}\left(|l|^{-\theta}+\left|U_{2}(z)\right|^{-\theta}\right)^{-\frac{1}{\theta}-1} \frac{1}{f_{1}\left(U_{1}^{-1}(l)\right)} d l\right| .
\end{aligned}
$$

Observe, we have

$$
U_{1}^{-1}(l)=\beta^{\frac{1}{\beta}} l^{-1 / \beta} .
$$

Since $f_{1}(y)=y^{-1-\beta}$ we get for any $\gamma>0$

$$
g(l):=\frac{1}{f_{1}\left(U_{1}^{-1}(l)\right)}=C_{\beta} l^{-\frac{1}{\beta}-1} .
$$

Thus, we can write

$$
\left|I_{1}(z)\right| \leq 2(1+\theta) C_{\beta}|\xi| \int_{0}^{\infty}\left(\left(\frac{|l|}{\left|U_{2}(z)\right|}\right)^{-\theta}+1\right)^{-\frac{1}{\theta}-1} l^{-\frac{1}{\beta}-1} d l
$$


Substitution gives

$$
\begin{aligned}
\left|I_{1}(z)\right| & \leq 2(1+\theta) C_{\beta}|\xi| \int_{0}^{\infty}\left(u^{-\theta}+1\right)^{-\frac{1}{\theta}-1}\left(u U_{2}(z)\right)^{-\frac{1}{\beta}-1} U_{2}(z) d u \\
& \leq 2(1+\theta) C_{\beta}|\xi|\left|U_{2}(z)\right|^{-\frac{1}{\beta}} \int_{0}^{\infty}\left(u^{-\theta}+1\right)^{-\frac{1}{\theta}-1} u^{-\frac{1}{\beta}-1} d u .
\end{aligned}
$$

Now consider,

$$
\begin{aligned}
\left|I_{2}(z)\right| \leq & 2(1+\theta)\left|U_{2}(z)\right|^{-\theta-1} \\
& \times\left.\left|\int_{0}^{\infty} i \xi y\left(\left|U_{1}(y)\right|^{-\theta}+\left|U_{2}(z)\right|^{-\theta}\right)^{-\frac{1}{\theta}-2}\right| U_{1}(y)\right|^{-\theta-1} f_{1}(y) d y \mid .
\end{aligned}
$$

Substitution with $v=U_{1}(y)$ gives the estimate

$$
\begin{aligned}
\left|I_{2}(z)\right| \leq & 2(1+\theta) \beta^{\frac{1}{\beta}}\left|U_{2}(z)\right|^{-\theta-1}|\xi| \\
& \times\left.\left|\int_{0}^{\infty}\left(|v|^{-\theta}+\left|U_{2}(z)\right|^{-\theta}\right)^{-\frac{1}{\theta}-2}\right| v\right|^{-\theta-\frac{1}{\beta}-1} d v \mid \\
= & 2(1+\theta) \beta^{\frac{1}{\beta}}\left|U_{2}(z)\right|^{\theta}|\xi| \\
& \times\left.\left|\int_{0}^{\infty}\left(\left(\frac{|v|}{\left|U_{2}(z)\right|}\right)^{-\theta}+1\right)^{-\frac{1}{\theta}-2}\right| v\right|^{-\theta-\frac{1}{\beta}-1} d v \mid .
\end{aligned}
$$

Now take $u=\frac{|v|}{\left|U_{2}(z)\right|}$ as a substitution to obtain,

$$
\begin{aligned}
\left|I_{2}(z)\right| \leq & 2(1+\theta) \beta^{\frac{1}{\beta}}\left|U_{2}(z)\right|^{-\frac{1}{\beta}}|\xi| \\
& \times \int_{0}^{\infty}\left(u^{-\theta}+1\right)^{-\frac{1}{\theta}-2} u^{-\theta-\frac{1}{\beta}-1} d u .
\end{aligned}
$$

Since $U_{2}(z)=\beta|z|^{-\beta}$ we have

$$
\left|I_{1}(z)+I_{2}(z)\right| \leq 2 \hat{C}|\xi||z|,
$$

where

$$
\begin{aligned}
\hat{C}= & 2(1+\theta)\left(C_{\beta} \int_{0}^{\infty}\left(u^{-\theta}+1\right)^{-\frac{1}{\theta}-1} u^{-\frac{1}{\beta}-1} d u\right. \\
& \left.+\beta^{\frac{1}{\beta}} \int_{0}^{\infty}\left(u^{-\theta}+1\right)^{-\frac{1}{\theta}-2} u^{-\theta-\frac{1}{\beta}-1} d u\right) .
\end{aligned}
$$

Since $\beta \leq 1$, we have

$$
\int_{|z| \leq 1} k_{2}(z)^{p} v_{2}(d z)=\int_{-1}^{1}|z|^{p}|z|^{-\beta-1} d z<\infty,
$$


for any $p>1$. This shows that the upper index of $\Theta_{z}$ is 1 . Since $\alpha>1$, there exists a number $p>1$ such that

$$
\frac{\beta^{+}}{\alpha^{-}}<\frac{1}{p} .
$$

By the assumptions, the law of $X_{0}$ has a density function $F$ which is integrable and $\rho_{0}(f)=\int_{\mathbb{R}} \rho_{0}(x) f(x) d x$. Therefore, we have $\rho_{0} \in L^{2}(\mathbb{R})$. Hence, by Theorem 3.1 one can conclude the proof of Corollary 3.1.

\section{Appendix A: The Zakai equation as a stochastic evolution equation}

In this appendix, we treat the Zakai equation as a stochastic evolution equation on a Hilbert space and establish the existence and uniqueness of its mild solution. For doing so, let $\mathfrak{X}$ be a Hilbert space, $A$ be a possibly unbounded operator generating an analytic $C_{0}$ semigroup $\left(T_{A}(t)\right)_{t \geq 0}$ on $\mathfrak{X}$. Let $\eta$ be a time homogenous Poisson random measure with Lévy measure $v$ on a measurable space $(Z, \mathcal{Z})$ over a probability space $\left(\Omega, \mathcal{G},\left(\mathcal{G}_{t}\right)_{t \geq 0}, \mathbb{Q}\right)$ and $B=\{B(t): t \geq 0\}$ be a 1-dimensional Brownian motion defined over the same filtered probability space. Let $f: \mathfrak{X} \rightarrow \mathfrak{X}$, $\Sigma: \mathfrak{X} \rightarrow \mathfrak{X}$ be two mappings and $G:[0, T] \times \mathfrak{X} \times \mathbb{R} \rightarrow \mathfrak{X}$ be a progressively measurable mapping. Consider the following equation with random initial data $u_{0}$ :

$$
\left\{\begin{aligned}
& d u(t)=(A u(t)+f(u(t))) d t+\Sigma(u(t)) d B(t) \\
&+\int_{\mathbb{R}} G\left(t, u\left(t^{-}\right), z\right) \tilde{\eta}(d z, d t) \\
& u(0)=u_{0} \in \mathfrak{X}, \quad \mathbb{P} \text {-a.s. }
\end{aligned}\right.
$$

where $\tilde{\eta}(d z, d t)=\eta(d z, d t)-v(d z) d t$ is the compensated Poisson random measure. Now we define the concept of solution we have in mind.

Definition A.1. We call a stochastic process $u=\{u(t): t \geq 0\}$ a mild solution to (A.1), if $u$ is càdlàg in $\mathfrak{X}$ and satisfies $\mathbb{P}$-a.s.

$$
\begin{aligned}
u(t)= & u_{0}+\int_{0}^{t} T_{A}(t-r) f(u(r)) d r \\
& +\int_{0}^{t} T_{A}(t-r) \Sigma(u(r)) d B(r)+\int_{0}^{t} \int_{\mathbb{R}} T_{A}(t-r) G\left(r, u\left(r^{-}\right), z\right) \tilde{\eta}(d z, d r) .
\end{aligned}
$$

We state and prove the following result.

Theorem A.1. Fix $\varrho \in \mathbb{R}$. Let us assume that

- there exists some $\varrho_{0}>-1$ such that $u_{0} \in H_{2}^{\varrho_{0}}(\mathbb{R}), \mathbb{P}$ a.s.;

- the operator A has symbol $\psi$ with lower Blumenthal-Getoor index $\alpha_{0}^{-}$; 
- there exists a $\delta_{f}<\alpha_{0}^{-}$and a constant $C_{f}>0$ with

$$
|f(x)-f(y)|_{H_{2}^{\varrho-\delta_{f}}} \leq C_{f}|x-y|_{H_{2}^{\varrho}}, \quad x, y \in H_{2}^{\varrho}(\mathbb{R}) ;
$$

- there exists a $\delta_{\Sigma}<\frac{\alpha_{0}^{-}}{2}$ and a constant $C_{\Sigma}>0$ such that

$$
|\Sigma(x)-\Sigma(y)|_{H_{2}^{\varrho-\delta_{\Sigma}}} \leq C_{\Sigma}|x-y|_{H_{2}^{\varrho}}, \quad x, y \in H_{2}^{\varrho}(\mathbb{R}) ;
$$

- there exists $\beta^{+} \geq 0$ and some $q \in[1,2]$ such that the operator $G$ satisfies the following inequality

$$
\int_{|z| \leq 1}|G(r, x, z)-G(r, y, z)|_{H_{2}^{\varrho-\beta^{+}}(\mathbb{R})}^{q} v(d z) \leq C_{G}|x-y|_{H_{2}^{\varrho}}^{q},
$$

$y, x \in H_{2}^{\varrho}(\mathbb{R})$ and for $|z| \geq 1, r \in[0, T]$,

$$
\begin{aligned}
& |G(r, x, z)-G(r, x, z)|_{H_{2}^{\rho-\beta^{+}}(\mathbb{R})} \\
& \quad \leq C_{G}|x-y|_{H_{2}^{\rho}}, \quad y, x \in H_{2}^{\varrho}(\mathbb{R}), r \in[0, T] .
\end{aligned}
$$

In addition, if

$$
\varrho-\varrho_{0}<\frac{1}{q} \text { and } \quad \frac{\beta^{+}}{\alpha_{0}^{-}}<\frac{1}{q},
$$

then, there exists a mild solution $u$ belonging $\mathbb{P}$-a.s. to $\mathbb{D}\left((0, T], H_{2}^{\varrho}(\mathbb{R})\right) \cap$ $\mathbb{D}\left([0, T] ; H_{2}^{\varrho_{0}}(\mathbb{R})\right)$ of the stochastic evolution equation

$$
\left\{\begin{aligned}
d u(t)= & (A u(t)+f(u(t))) d t+\Sigma(u(t)) d B(t) \\
& +\int_{\mathbb{R}} G\left(t, u\left(t^{-}\right), z\right) \tilde{\eta}(d z, d t), \\
u(0)= & u_{0}, \quad \mathbb{P} \text {-a.s. }
\end{aligned}\right.
$$

with random initial data $u_{0} \in H_{2}^{\rho_{0}}(\mathbb{R})$.

Proof. First, we tackle the case where the $q$-moments are bounded, that is, we suppose

$$
\int|G(r, x, z)-G(r, y, z)|_{H_{2}^{\rho-\beta^{+}}(\mathbb{R})}^{q} v(d z) \leq C_{G}|x-y|_{H_{2}^{\varrho}}^{q},
$$

for $y, x \in H_{2}^{\varrho}(\mathbb{R}), r \in[0, T]$. Let $\varrho<\varrho_{0}+\frac{1}{q}$ and

$$
\begin{aligned}
\mathbb{M}_{\lambda, \varrho}^{q}([0, T] \times \Omega ; \mathbb{R}):= & \{u:[0, T] \times \Omega \rightarrow \mathbb{R}, u \text { is progressively measurable } \\
& \text { and } \left.\mathbb{E} \int_{0}^{T} e^{-\lambda t}|u(t)|_{H_{2}^{\varrho}(\mathbb{R})}^{q} d t<\infty\right\}
\end{aligned}
$$


equipped with the norm

$$
|u|_{\mathbb{M}_{\lambda, \varrho}^{q}}:=\left(\mathbb{E} \int_{0}^{T} e^{-\lambda t}|u(t)|_{H_{2}^{\varrho}(\mathbb{R})}^{q} d t\right)^{\frac{1}{q}}, \quad u \in \mathbb{M}_{\lambda, \varrho}^{q}([0, T] \times \Omega ; \mathbb{R}) .
$$

Now, the existence of the mild solution will be established by making use of Banach fixed point theorem (see, e.g., Hausenblas (2005)). For any $\lambda>0$ let us define the operator

$$
\mathcal{I}: \mathbb{M}_{\lambda, \varrho}^{q}([0, T] \times \Omega ; \mathbb{R}) \rightarrow \mathbb{M}_{\lambda, \varrho}^{q}([0, T] \times \Omega ; \mathbb{R})
$$

by

$$
\begin{aligned}
\mathcal{I}(u)(t)= & T_{A}(t) u_{0}+\int_{0}^{t} T_{A}(t-r) f(u(r)) d r+\int_{0}^{t} T_{A}(t-r) \Sigma(u(r)) d B(r) \\
& +\int_{0}^{t} \int_{|z| \leq 1} T_{A}(t-r) G\left(r, u\left(r^{-}\right), z\right) \tilde{\eta}(d z, d r), \quad t \geq 0,
\end{aligned}
$$

and $u \in \mathbb{M}_{\lambda, \varrho}^{q}([0, T] \times \Omega ; \mathbb{R})$. First, we have to show that $\mathcal{I}$ maps $\mathbb{M}_{\lambda, \varrho}^{q}([0, T] \times$ $\Omega ; \mathbb{R}$ ) into itself. Since the symbol of $\psi_{0}$ has a Blumenthal-Getoor lower index $\alpha_{0}^{-}$, Theorem 2.1 in Fernando Pani, Hausenblas and Razafimandimby (2017) implies that for $\gamma \geq 0$ and $\delta \in \mathbb{R}$

$$
\left|T_{A}(t) u_{0}\right|_{H_{2}^{\delta-\gamma}(\mathbb{R})} \leq C t^{-\frac{\gamma}{\alpha_{0}^{-}}}\left|u_{0}\right|_{H_{2}^{\delta}(\mathbb{R})}, \quad u_{0} \in H_{2}^{\delta}(\mathbb{R}) .
$$

Hence,

$$
\begin{aligned}
& \mathbb{E} \int_{0}^{T} e^{-\lambda r}\left|T_{A}(r) u_{0}\right|_{H_{2}^{\varrho}}^{q} d r \\
& \quad \leq C \mathbb{E} \int_{0}^{T} e^{-\lambda r} r^{-\frac{q\left(\rho-\rho_{0}\right)}{\alpha_{0}^{-}}}\left|u_{0}\right|_{H_{2}^{\rho_{0}}}^{q} d r \leq \frac{C}{\lambda^{1-\frac{q\left(\rho-\rho_{0}\right)}{\alpha_{0}^{-}}}} \mathbb{E}\left|u_{0}\right|_{H_{2}^{\varrho}}^{\varrho} .
\end{aligned}
$$

The Minkowski's integral inequality and the assumption regarding on $f$ give for the second term

$$
\begin{aligned}
\mathbb{E} \int_{0}^{T} & e^{-\lambda t}\left|\int_{0}^{t} T_{A}(t-r) f(u(r)) d r\right|_{H_{2}^{\varrho}}^{q} d t \\
& \leq \int_{0}^{T} e^{-\lambda t} \mathbb{E}\left(\int_{0}^{t}\left|T_{A}(t-r) f(u(r))\right|_{H_{2}^{\varrho}} d r\right)^{q} d t \\
& \leq C^{q} \int_{0}^{T} \mathbb{E}\left(\int_{0}^{t} e^{-\frac{\lambda(t-r)}{q}}(t-r)^{-\frac{\delta_{f}}{\alpha_{0}^{-}}} e^{-\frac{\lambda r}{q}}|f(u(r))|_{H_{2}^{\varrho-\delta_{f}}} d r\right)^{q} d t \\
& \leq\left(C C_{f}\right)^{q} \int_{0}^{T} \mathbb{E}\left(\int_{0}^{t} e^{-\frac{\lambda(t-r)}{q}}(t-r)^{-\frac{\delta_{f}}{\alpha_{0}^{-}}} e^{-\frac{\lambda r}{q}}\left(1+|u(r)|_{H_{2}^{\varrho}}\right) d r\right)^{q} d t .
\end{aligned}
$$


Applying Young's inequality for the convolution term gives

$$
\begin{aligned}
\mathbb{E} \int_{0}^{T} e^{-\lambda t}\left|\int_{0}^{t} T_{A}(t-r) f(u(r)) d r\right|_{H_{2}^{\varrho}}^{q} d t \\
\leq\left(C C_{f}\right)^{q} \int_{0}^{T} e^{-\frac{\lambda r}{q}} r^{-\frac{\delta_{f}}{\alpha_{0}^{-}}} d r \cdot \int_{0}^{T} e^{-\lambda r} \mathbb{E}\left(1+|u(r)|_{H_{2}}\right)^{q} d r \\
\leq \frac{C_{1}}{\lambda^{1-\frac{\delta_{f}}{\alpha_{0}^{-}}}} \cdot \int_{0}^{T} e^{-\lambda r} \mathbb{E}\left(1+|u(r)|_{H_{2}^{\varrho}}\right)^{q} d r<\infty,
\end{aligned}
$$

where $C_{1}=\left(C C_{f}\right)^{q} q^{1-\frac{\delta_{f}}{\alpha_{0}^{-}}} \int_{0}^{\frac{\lambda T}{q}} e^{-\theta} \theta^{-\frac{\delta_{f}}{\alpha_{0}^{-}}} d \theta$. For the third term, we get

$$
\begin{aligned}
\mathbb{E} \int_{0}^{T} & {\left[\int_{0}^{t}\left|e^{-\frac{\lambda(t-r)}{q}} T_{A}(t-r) e^{-\frac{\lambda r}{q}} \Sigma(u(r))\right|_{H_{2}^{\varrho}}^{2} d r\right]^{\frac{q}{2}} d t } \\
& \leq C^{q} \mathbb{E} \int_{0}^{T}\left[\int_{0}^{t}(t-r)^{-\frac{2 \delta_{\Sigma}}{\alpha_{0}^{-}}} e^{-\frac{2 \lambda(t-r)}{q}} e^{\left.-\frac{2 \lambda r}{q}|\Sigma(u(r))|^{2}{ }_{H_{2}}{ }_{-\frac{\delta_{\Sigma}}{\alpha_{0}^{-}}} d r\right]^{\frac{q}{2}} d t .}\right.
\end{aligned}
$$

By the assumption on $\Sigma$, we can infer that

$$
\cdots \leq\left(C C_{\Sigma}\right)^{q} \mathbb{E} \int_{0}^{T}\left[\int_{0}^{t}(t-r)^{-\frac{2 \delta_{\Sigma}}{\alpha_{0}^{-}}} e^{-\frac{2 \lambda(t-r)}{q}} e^{-\frac{2 \lambda r}{q}}\left(1+|u(r)|_{\left.H_{2}^{\varrho}\right)^{2}} d r\right]^{\frac{q}{2}} d t .\right.
$$

Then applying Young's inequality for the convolution

$$
\cdots \leq\left(C C_{\Sigma}\right)^{q} \int_{0}^{T} r^{-\frac{2 \delta_{\Sigma}}{\alpha_{0}^{-}}} e^{-\frac{2 \lambda r}{q}} d r \cdot \int_{0}^{T} e^{-\lambda r} \mathbb{E}\left(1+|u(r)|_{H_{2}^{\varrho}}\right)^{q} d r .
$$

Hence, we have

$$
\begin{gathered}
\mathbb{E} \int_{0}^{T} e^{-\lambda t}\left|\int_{0}^{t} T_{A}(t-r) \Sigma(u(r)) d W(r)\right|_{H_{2}^{\varrho}}^{q} d t \\
\quad \leq \frac{C_{2}}{\lambda^{1-\frac{2 \delta_{\Sigma}}{\alpha_{0}^{-}}}} \int_{0}^{T} e^{-\lambda t} \mathbb{E}(1+|u(t)|)_{H_{2}^{\varrho}}^{q} d t<\infty,
\end{gathered}
$$

where $C_{2}=\left(C C_{\Sigma}\right)^{q}(q / 2)^{1-\frac{2 \delta_{\Sigma}}{\alpha_{0}^{-}}} \int_{0}^{\frac{2 \lambda T}{q}} e^{-\theta} \theta^{-\frac{\delta_{\Sigma}}{\alpha_{0}^{-}}} d \theta$. It remains to calculate the fourth term. By the assumptions on $G$ and $A$, we get

$$
\begin{aligned}
\int_{\mathbb{R}} \mid & \left.T_{A}(t) G(r, x, z)\right|_{H_{2}^{\rho}\left(\mathbb{R}^{d}\right)} ^{q} v(d z) \\
& \leq C^{q} t^{-q \frac{\beta^{+}}{\alpha_{0}^{-}}}\left(1+|x|_{H_{2}^{\varrho}}\right)^{q}, \quad x \in H_{2}^{\varrho}(\mathbb{R}) .
\end{aligned}
$$


In particular, if

$$
2 \frac{\beta^{+}}{\alpha_{0}^{-}}<1
$$

one can deal with the fourth term as follows. Consider first,

$$
\begin{aligned}
\mathbb{E} \int_{0}^{T} & {\left[\int_{0}^{t} \int_{\mathbb{R}}\left|e^{-\frac{\lambda(t-r)}{q}} T_{A}(t-r) e^{-\frac{\lambda r}{q}} G(r, u(r), z)\right|_{H_{2}^{\varrho}}^{2} v(d z) d r\right]^{\frac{q}{2}} d t } \\
& \leq\left(C C_{G}\right)^{q} \mathbb{E} \int_{0}^{T}\left[\int_{0}^{t}(t-r)^{-\frac{2 \beta^{+}}{\alpha_{0}^{-}}} e^{-\frac{2 \lambda(t-r)}{q}} e^{-\frac{2 \lambda r}{q}}\left(1+|u(r)|_{H_{2}}\right)^{2} d r\right]^{\frac{q}{2}} d t .
\end{aligned}
$$

By following similar argument as in Brownian term, we have

$$
\begin{gathered}
\mathbb{E} \int_{0}^{T} e^{-\lambda t}\left|\int_{0}^{t} \int_{\mathbb{R}} T_{A}(t-r) G\left(r, u\left(r^{-}\right), z\right) \tilde{\eta}(d z, d r)\right|_{H_{2}^{\varrho}}^{q} d t \\
\leq \frac{C_{3}}{\lambda^{1-\frac{2 \beta^{+}}{\alpha_{0}^{-}}}} \int_{0}^{T} e^{-\lambda t} \mathbb{E}(1+|u(t)|)_{H_{2}^{\varrho}}^{q} d t<\infty
\end{gathered}
$$

where $C_{3}=\left(C C_{G}\right)^{q}(q / 2)^{1-\frac{2 \beta^{+}}{\alpha_{0}^{-}}} \int_{0}^{\frac{2 \lambda T}{q}} e^{-\theta} \theta^{-\frac{\beta^{+}}{\alpha_{0}^{-}}} d \theta$. Then collecting all estimates yields that $\mathcal{I}$ maps $\mathbb{M}_{\lambda, \varrho}^{q}([0, T] \times \Omega ; \mathbb{R})$ into itself.

Next, we will show that there exists a $\lambda>0$ such that the operator $\mathcal{I}$ : $\mathbb{M}_{\lambda, \varrho}^{q}([0, T] \times \Omega ; \mathbb{R}) \rightarrow \mathbb{M}_{\lambda, \varrho}^{q}([0, T] \times \Omega ; \mathbb{R})$ is a strict contraction. To show the claim, let $u, v \in \mathbb{M}_{\lambda, \varrho}^{q}([0, T] \times \Omega ; \mathbb{R})$. Then

$$
\begin{aligned}
\mid \mathcal{I}(u)- & \left.\mathcal{I}(v)\right|_{\mathbb{M}_{\lambda, \varrho}^{q}} ^{q} \\
\leq & \int_{0}^{T} e^{-t \lambda} \mathbb{E}\left|\int_{0}^{t} T_{A}(t-r)(\Sigma(u(r))-\Sigma(v(r))) d B(r)\right|_{H_{2}^{\varrho}}^{q} d t \\
& +\int_{0}^{T} e^{-t \lambda} \mathbb{E} \mid \int_{0}^{t} \int_{\mathbb{R}} T_{A}(t-r)\left(G\left(r, u\left(r^{-}\right), z\right)\right. \\
& \left.-G\left(r, v\left(r^{-}\right), z\right)\right)\left.\eta(d z, d r)\right|_{H_{2}^{\varrho}} ^{q} d t \\
& +\int_{0}^{T} e^{-t \lambda} \mathbb{E}\left|\int_{0}^{t} T_{A}(t-r)(f(u(r))-f(v(r))) d r\right|_{H_{2}^{\varrho}}^{q} d t .
\end{aligned}
$$

Then by following similar arguments as in previous calculation, we can easily show that,

$$
|\mathcal{I}(u)-\mathcal{I}(v)|_{\mathbb{M}_{\lambda, \varrho}^{q}}^{q} \leq \frac{\hat{C}}{\lambda^{\varepsilon}}|u-v|_{\mathbb{M}_{\lambda, \varrho}^{q}}^{q}
$$


where $\hat{C}=\max \left\{C_{1}, C_{2}, C_{3}\right\}$ and $\varepsilon=\min \left\{1-\frac{\delta_{f}}{\alpha_{0}^{-}}, 1-\frac{2 \delta_{\Sigma}}{\alpha_{0}^{-}}, 1-\frac{2 \beta^{+}}{\alpha_{0}^{-}}\right\}$. Hence $\mathcal{I}$ is a strict contraction for $\lambda$ sufficiently large.

To conclude the proof of the theorem we show that $u \in \mathbb{D}\left((0, T], H_{2}^{\varrho}(\mathbb{R})\right) \cap$ $\mathbb{D}\left([0, T] ; H_{2}^{\varrho 0}(\mathbb{R})\right)$. For this purpose, we consider the stochastic convolution term with respect to the Brownian term, that is,

$$
\int_{0}^{t} T_{A}(t-r) \Sigma(u(r)) d B(r) .
$$

The continuity of this term follows by DaPrato and Zabczyk (1992), Theorem 5.9, page 127 . It remains to investigate the càdlàg property of

$$
\int_{0}^{t} \int_{\mathbb{R}} T_{A}(t-r) G\left(r, u\left(r^{-}\right), z\right) \tilde{\eta}(d z, d r) .
$$

But Proposition 1.3 in Hausenblas and Seidler (2008) leads to

$$
\begin{gathered}
\mathbb{E}\left|\int_{0}^{t} \int_{\mathbb{R}} T_{A}(t-r) G\left(r, u\left(r^{-}\right), z\right) \tilde{\eta}(d z, d r)\right|_{H_{2}^{\varrho-\beta^{+}}}^{q} \\
\leq \mathbb{E} \int_{0}^{t} \int_{\mathbb{R}}|G(r, u(r), z)|_{H_{2}^{\varrho-\beta^{+}}}^{q} v(d z) d r .
\end{gathered}
$$

Since for any $z \in \mathbb{R}_{+}^{0}, G(\cdot, \cdot, z): H_{2}^{\varrho}(\mathbb{R}) \rightarrow H_{2}^{\varrho-\beta^{+}}(\mathbb{R})$ is bounded, the càdlàg property follows.

In previous analysis, we assumed that $q$-moments are bounded of the jump term (see (A.5)) to construct the solution to (A.4) using fixed point method. In general, we should only consider small jumps with the assumption (A.5) and prove the existence of the solution by using fixed point method, since if we allow large jumps to occur, then the corresponding jump integral may blow up and the fixed point method will collapse. Notice that the random jump times with jump size larger than one are independent of the $\sigma$-algebra generated by small jumps (size less than one) and Brownian motion. In particular, the Poisson random measure is independently scattered, or in other words, for any $U \in \mathcal{B}(\mathbb{R})$ the processes $\eta(U \cap(-1,1) \times[0, t])$ and $\eta(U \cap \mathbb{R} \backslash(-1,1) \times[0, t])$ are independent. Therefore, now we assume that (A.5) holds with only small jumps (size less than one). Let $\left\{T_{i}: i=1, \ldots, n\right\}$ be the random jump times (stopping times) with the size of the jumps are larger than one. Previous analysis guarantees that there exists a $\hat{u} \in \mathbb{D}\left(\left(0, T_{1}\right), H_{2}^{\varrho}(\mathbb{R})\right) \cap$ $\mathbb{D}\left(\left[0, T_{1}\right) ; H_{2}^{\varrho 0}(\mathbb{R})\right)$, which solves

$$
\left\{\begin{aligned}
& d \hat{u}(t)=(A \hat{u}(t)+f(\hat{u}(t))) d t+\Sigma(\hat{u}(t)) d B(t) \\
&+\int_{|z|<1} G\left(t, \hat{u}\left(t^{-}\right), z\right) \tilde{\eta}(d z, d t) \\
&-\int_{|z| \geq 1} G(t, \hat{u}(t), z) v(d z) d t \\
& \hat{u}(0)=\hat{u}_{0} \in \mathfrak{X}, \quad \mathbb{P} \text {-a.s. }
\end{aligned}\right.
$$


We follow interlacing criteria (see Theorem 2.5.1 in Applebaum (2009)) to construct the solution over whole interval $[0, T]$.

Now we recursively construct the solution $u=u_{n}$ of (A.4) over whole interval $[0, T]$ as follows. Define on $\left[0, T_{1}\right]$

$$
u_{1}(t)= \begin{cases}\hat{u}(t) & \text { for } t<T_{1}, \\ \hat{u}\left(T_{1}^{-}\right)+G\left(T_{1}^{-}, \hat{u}\left(T_{1}^{-}\right), \Delta P\left(T_{1}\right)\right) & \text { for } t=T_{1},\end{cases}
$$

where $P(t)=\int_{|z| \geq 1} z \eta(d z, d t)$ is the compound Poisson process. Now suppose that $\mathbb{P}\left\{\omega \in \Omega: T_{1}<\infty\right\}=1$. Define $\bar{u}(0)=u_{1}\left(T_{1}\right), \bar{B}(t)=B\left(T_{1}+t\right), \bar{\eta}(\cdot, t)=$ $\eta\left(\cdot, T_{1}+t\right)$ and $\overline{\mathscr{F}}_{t}=\mathscr{F}_{T_{1}+t}$. Let $\bar{P}(t)=\int_{|z| \geq 1} z \bar{\eta}(d z, d t)$ be the compound Poisson process which starts from time $T_{1}$.

Since we don't have jumps with size larger than one during the time interval $\left(T_{1}, T_{2}\right)$, from previous analysis there exists a solution $\bar{u}\left(t-T_{1}\right) \in$ $\mathbb{D}\left(\left(T_{1}, T_{2}\right), H_{2}^{\varrho}(\mathbb{R})\right) \cap \mathbb{D}\left(\left[T_{1}, T_{2}\right) ; H_{2}^{\varrho 0}(\mathbb{R})\right)$. Then,

$u_{2}(t)$

$$
= \begin{cases}u_{1}(t) & \text { for } t \leq T_{1}, \\ \bar{u}\left(t-T_{1}\right) & \text { for } T_{1} \leq t \leq T_{2}, \text { (A.10) } \\ \bar{u}\left(\left(T_{2}-T_{1}\right)^{-}\right) & \\ \quad+G\left(\left(T_{2}-T_{1}\right)^{-}, \bar{u}\left(\left(T_{2}-T_{1}\right)^{-}\right), \Delta \bar{P}\left(T_{2}\right)\right) & \text { for } t=T_{2} .\end{cases}
$$

Since we have a finite number of large jumps with size bigger than one over $[0, T]$ almost surely, by repeating the above process $n$ times, we can obtain $u=u_{n} \in$ $\mathbb{D}\left((0, T], H_{2}^{\varrho}(\mathbb{R})\right) \cap \mathbb{D}\left([0, T] ; H_{2}^{\varrho_{0}}(\mathbb{R})\right)$ which solves (A.4).

\section{Appendix B: Lévy copulas}

Lévy copulas is a general concept to capture jump dependency in multivariate Lévy processes and is widely used in finance. In this section, we only recall short facts about copulas, pair copulas, Lévy processes, and the Lévy copula concept. Detailed treatment of copulas and Lévy copulas can be found in Cherubini, Luciano and Vecchiato (2004), Nelsen (2006), Malevergne and Sornette (2006) and Cont and Tankov (2004, 2006), Kallsen and Tankov (2006).

Let $L_{1}$ and $L_{2}$ be two Lévy processes with Lévy measures $v_{1}$ and $v_{2}$. Before introducing the Lévy copulas, let us introduce the extended tail integrals $U_{1}$ and $U_{2}$.

First, we need following function associated with any $z \in \mathbb{R} \backslash\{0\}$ :

$$
\mathcal{I}(z)= \begin{cases}(z, \infty), & z>0 \\ (-\infty, z), & z<0\end{cases}
$$


In the same way as the distribution of a random vector can be represented by its distribution function, the Lévy measure of a Lévy process will be represented by its tail integral.

Now, the tail integral of a 2-dimensional process can be defined for $i=1,2$ by

$$
U_{i}(z)= \begin{cases}\operatorname{sgn}(z) v_{i}(\mathcal{I}(z)) & \text { if } z \in \mathbb{R} \backslash\{0\}, \\ 0 & \text { if } z=\infty\end{cases}
$$

and their generalized inverse, given by

$$
U_{i}^{\leftarrow}(z):=\sup \left\{x \geq 0 \mid U_{i}(x)=z\right\}, \quad z \geq 0, i=1,2 .
$$

Dependence of jumps of a multivariate Lévy process can be described by a Lévy copula which couples the marginal tail integrals. In particular, let $L$ be a two dimensional Lévy process, $v$ is its intensity measure and $U$ is the tail integral defined by

$$
U(z)=\prod_{i=1}^{2} \operatorname{sgn}\left(z_{i}\right) \nu\left(\prod_{i=1}^{2} \mathcal{I}\left(z_{i}\right)\right), \quad z=\left(z_{1}, z_{2}\right) \in(\mathbb{R} \backslash\{0\} \cup\{\infty\})^{2} .
$$

Now, $L$ can be seen as two Lévy processes linked together by the mapping $H$ : $\mathbb{R}^{2} \rightarrow \mathbb{R}$, defined as

$$
U\left(z_{1}, z_{2}\right)=H\left(U_{1}\left(z_{1}\right), U_{2}\left(z_{2}\right)\right), \quad z_{1}, z_{2} \in \mathbb{R} \backslash\{0\} \cup\{\infty\} .
$$

For example, if $L_{1}$ and $L_{2}$ are independent positive Lévy processes, the copula $H$ is given by (see Kallsen and Tankov (2006), Theorem 4.6)

$$
H_{\perp}\left(z_{1}, z_{2}\right)=z_{1} 1_{z_{2}=\infty}+z_{2} 1_{z_{1}=\infty}, \quad z_{1}, z_{2} \in \mathbb{R}^{+} \cup\{\infty\} .
$$

If $L_{1}$ and $L_{2}$ are completely dependent, the copula $H$ is given by

$$
H_{\|}\left(z_{1}, z_{2}\right)=\min \left(\left|z_{1}\right|,\left|z_{2}\right|\right) 1_{K}\left(z_{1}, z_{2}\right) \operatorname{sgn}\left(z_{1}\right) \operatorname{sgn}\left(z_{2}\right), \quad z_{1}, z_{2} \in \mathbb{R},
$$

where $K=\left\{\left(z_{1}, z_{2}\right) \in \mathbb{R}^{2}: \operatorname{sgn}\left(z_{1}\right)=\operatorname{sgn}\left(z_{2}\right)\right\}$.

A Sklar type Theorem (see Cont and Tankov (2004)) ensures the existence and uniqueness of a Lévy copula given a Lévy process, and vice versa. To be more precise, it says that for each 2-dimensional Lévy process with intensity $v$ and marginal Lévy measures $v_{i}, i=1,2$, one can associate a Lévy copula $H$ such that

$$
\begin{aligned}
U\left(z_{1}, z_{2}\right)= & \operatorname{sgn}\left(z_{1}\right) \operatorname{sgn}\left(z_{2}\right) \\
& \times H\left(U_{1}\left(z_{1}\right), U_{2}\left(z_{2}\right)\right), \quad z_{1}, z_{2} \in \mathbb{R} \backslash\{0\} \cup\{\infty\} .
\end{aligned}
$$

Here $U$ and $U_{i}, i=1,2$, denotes the tail integrals defined by (2.2) and (2.1) respectively.

Conversely, if $H$ is a Lévy copula and $U_{1}, U_{2}$ are marginal tail integrals of two Lévy processes, Equation B.3 defines the tail integral of a 2-dimensional Lévy process, where $U_{1}, U_{2}$ are the tail integrals of its components.

As an example, let us consider Clayton Lévy copula. 
Example B.1. For a 2-dimensional Lévy processes the Clayton copula is given on $\mathbb{R}^{2}$ by (see e.g. Cont and Tankov (2006), Kallsen and Tankov (2006))

$$
\begin{aligned}
H\left(z_{1}, z_{2}\right)= & \left(\frac{1}{2}\left|z_{1}\right|^{-\theta}+\frac{1}{2}\left|z_{2}\right|^{-\theta}\right)^{-\frac{1}{\theta}} \\
& \times\left(\beta 1_{z_{1} \cdot z_{2}>0}+(1-\beta) 1_{z_{1} \cdot z_{2}<0}\right), \quad z_{1}, z_{2} \in \mathbb{R} .
\end{aligned}
$$

The parameter $\theta>0$ determines the dependence of the jump sizes, where larger values of $\theta$ indicate a stronger dependence, smaller values of $\theta$ indicate independence. The parameter $\beta$ determines the dependence of the sign of jumps: when $\beta=1$, the two components always jump in the same direction, and when $\beta=0$, positive jumps in one component are accompanied by negative jumps in the other and vice versa. For intermediate values of $\beta$, positive jumps in one component can correspond to both positive and negative jumps in the other component. The parameter $\theta$ is responsible for the dependence of absolute values of jumps in different components.

To give the connection between copulas and Lévy copulas let us define the survival copula. Let $F: \mathbb{R}^{2} \rightarrow[0,1]$ be a distribution function and $\bar{F}(x, y)=$ $1-F(x, y)$. Let $F_{1}$ and $F_{2}$ be the marginal distributions, $\bar{F}_{1}=1-F_{1}$ and $\bar{F}_{2}=1-F_{2}$ be the marginal tail functions respectively. Now, one can define the survival copula associated to $F$ by

$$
\bar{C}(u, v):=\bar{F}\left(\bar{F}_{1}^{-1}(u), \bar{F}_{1}^{-1}(v)\right), \quad(u, v) \in[0,1]^{2} .
$$

Since $C(u, 1)=u$ and $C(1, v)=v$, we get $\bar{C}(0, u)=u$ and $\bar{C}(v, 0)=v$.

\section{B.1 Finite Lévy measure and copula}

For simplicity, let $L=\left(L_{1}, L_{2}\right)$ be a two dimensional Lévy process with only positive jumps and with marginal Lévy measures $v_{1}, v_{2}$ and copula $H$. Here, we assume that $v_{1}$ and $v_{2}$ are two Lévy measures with $v_{1}((0, \infty))=\lambda_{1}$, $\nu_{2}((0, \infty))=\lambda_{2}$. We also assume that $H$ is twice differentiable and $\nu_{1}, v_{2}$ have densities with respect to Lebesgue measure on $\mathbb{R} \backslash\{0\}$. We will consider only copula, such that $L_{1}$ and $L_{2}$ have only common jumps.

Let $\left(\mathcal{F}_{t}^{1}\right)_{t \geq 0}$ be the filtration generated by $L_{1}$ and $\left(\mathcal{F}_{t}^{2}\right)_{t \geq 0}$ the filtration generated by $L_{2}$. We are interested in the jumps of $L_{1}$ given the jumps of $L_{2}$. Since

$$
v\left(\left(z_{1}, \infty\right),\left(z_{2}, \infty\right)\right)=H\left(U_{1}\left(z_{1}\right), U_{2}\left(z_{2}\right)\right)
$$

it follows that

$$
v\left(d z_{1}, d z_{2}\right)=\left.\frac{\partial^{2}}{\partial u_{1} \partial u_{2}} H\left(u_{1}, u_{2}\right)\right|_{\substack{u_{1}=U_{1}\left(z_{1}\right) \\ u_{2}=U_{2}\left(z_{2}\right)}} v_{1}\left(d z_{1}\right) v_{2}\left(d z_{2}\right) .
$$


Substitution gives

$$
\begin{aligned}
v(\mathbb{R} \times \mathbb{R}) & =\int_{0}^{\infty} \int_{0}^{\infty} v\left(d z_{1}, d z_{2}\right)=\int_{0}^{\lambda_{1}} \int_{0}^{\lambda_{2}} \frac{\partial^{2} H\left(u_{1}, u_{2}\right)}{\partial u_{1} \partial u_{2}} d u_{1} d u_{2} \\
& =H(0,0)-H\left(\lambda_{1}, 0\right)-H\left(0, \lambda_{2}\right)+H\left(\lambda_{1}, \lambda_{2}\right) \\
& =H\left(\lambda_{1}, \lambda_{2}\right):=\lambda_{H} .
\end{aligned}
$$

Since $v_{1}$ and $v_{2}$ are finite, it follows that $L(t)$ can be represented by the following sum

$$
L(t)=\sum_{n=1}^{N(t)} Y_{n}
$$

where $N=\{N(t): t \geq 0\}$ is a Poisson process with intensity $\lambda_{H}$ and $\left\{Y_{n}=\right.$ $\left.\left(Y_{n, 1}, Y_{n, 2}\right): n \in \mathbb{N}\right\}$ is a family of $\mathbb{R}^{2}$-valued independent random variables with distribution function $v / \lambda_{H}$. Calculating the Fourier transform one can easily see

$$
\begin{aligned}
\mathbb{E} e^{i x L(t)} & =\sum_{k=1}^{\infty} \mathbb{E}\left[e^{\sum_{n=1}^{k} i x Y_{n}} \mid N(t)=k\right] \mathbb{P}(N(t)=k) \\
& =\exp \left(-\lambda_{H} t\right) \sum_{k=1}^{\infty} \frac{\left(\lambda_{H} t\right)^{k}}{k !} \mathbb{E}\left[e^{i x Y_{1}}\right]^{k} \\
& =\exp \left(-\lambda_{H} t\right) \exp \left(t \int_{\mathbb{R}^{2}} e^{i x y} v(d x \times d y)\right) \\
& =\exp \left(t \int_{\mathbb{R}^{2}}\left(e^{i x y}-1\right) v(d x \times d y)\right) .
\end{aligned}
$$

We are interested in the conditional distribution of the jumps in the first variable, given the jumps in the second variable, that is, $Y_{n, 1}$, given the projection onto the second axis, that is, $Y_{n, 2}$.

If $\bar{C}$ is the survival copula of $Y_{n}$, that is,

$$
\bar{C}\left(u_{1}, u_{2}\right)=\bar{F}\left(\bar{F}_{1}^{-1}\left(u_{1}\right), \bar{F}_{2}^{-1}\left(u_{2}\right)\right), \quad u_{1}, u_{2} \in[0,1],
$$

with $\bar{F}_{i}\left(x_{i}\right)=U_{i}\left(x_{i}\right) / \lambda_{i}$, then

$$
\bar{C}\left(u_{1}, u_{2}\right)=\bar{F}\left(U_{1}^{-1}\left(\lambda_{1} u_{1}\right), U_{2}^{-1}\left(\lambda_{2} u_{2}\right)\right)=\frac{1}{\lambda_{H}} U\left(U_{1}^{-1}\left(\lambda_{1} u_{1}\right), U_{2}^{-1}\left(\lambda_{2} u_{2}\right)\right)
$$

and, by the definition of the Lévy copula $H$,

$$
\bar{C}\left(u_{1}, u_{2}\right)=\frac{1}{\lambda_{H}} H\left(\lambda_{1} u_{1}, \lambda_{2} u_{2}\right), \quad u_{1}, u_{2} \in[0,1] .
$$

Fix $\varepsilon>0$ and let us assume that we have a Lévy measure with infinite activity and that we cut of all jumps whose projection onto one of the two axis is smaller 
than $\varepsilon$. Then we have

$$
v((\varepsilon, \infty) \times(\varepsilon, \infty))=\left.H\left(U_{1}^{-1}(\varepsilon), U_{2}^{-1}(\varepsilon)\right)\right|_{\substack{u_{1}=U_{1}^{-1}(\varepsilon) \\ u_{2}=U_{2}^{-1}(\varepsilon)}}=\bar{C}(\varepsilon, \varepsilon) .
$$

This gives us the scaling property

$$
\frac{1}{\lambda} H\left(\lambda u_{1}, \lambda u_{2}\right)=H\left(u_{1}, u_{2}\right), \quad u_{1}, u_{2} \in \mathbb{R} \backslash\{0\},
$$

for $\lambda=H(\varepsilon, \varepsilon)$.

Proposition B.1. Let us assume that $\lambda_{1}=\lambda_{2}=\lambda=H(\varepsilon, \varepsilon)$ and let us assume that the Copula $H$ satisfies the following scaling property:

$$
\frac{1}{\lambda} H\left(\lambda u_{1}, \lambda u_{2}\right)=H\left(u_{1}, u_{2}\right), \quad u_{1}, u_{2} \in \mathbb{R} \backslash\{0\}
$$

Let us define

$$
h\left(u_{1}, u_{2}\right):=\frac{\partial^{2}}{\partial u_{1} \partial u_{2}} H\left(u_{1}, u_{2}\right) .
$$

Then, the conditional probability of $\Delta_{t} L_{1}$ given $\Delta_{t} L_{2}$ is represented by

$$
\mathbb{P}\left(\Delta_{t} L_{1}=z_{1} \mid \Delta_{t} L_{2}=z_{2}\right)=h\left(u_{1}, u_{2}\right) \underset{\substack{u_{1}=U_{1}\left(z_{1}\right) \\ u_{2}=U_{2}\left(z_{2}\right)}}{ } v\left(d z_{1}\right) .
$$

Proof. The formula can be shown by direct calculations. In particular,

$$
\begin{aligned}
\mathbb{P}\left(\Delta_{t} L_{1}=z_{1} \mid \Delta_{t} L_{2}=z_{2}\right) & =\frac{\mathbb{P}\left(\left\{\Delta_{t} L_{1}=z_{1}\right\} \cap\left\{\Delta_{t} L_{2}=z_{2}\right\}\right)}{\mathbb{P}\left(\left\{\Delta_{t} L_{2}=z_{2}\right\}\right)} \\
& =\frac{\frac{\partial^{2}}{\partial z_{1} \partial z_{2}} \bar{F}\left(z_{1}, z_{2}\right)}{\frac{\partial}{\partial z_{2}} \bar{F}\left(0, z_{2}\right)}=\frac{\frac{\partial^{2}}{\partial z_{1} \partial z_{2}} \bar{F}\left(z_{1}, z_{2}\right)}{\frac{\partial}{\partial z_{2}} \bar{F}_{2}\left(z_{2}\right)} \\
& =\frac{\frac{\partial^{2}}{\partial z_{1} \partial z_{2}} \bar{C}\left(F_{1}\left(z_{1}\right), F_{2}\left(z_{2}\right)\right)}{\frac{\partial}{\partial z_{2}} \bar{F}\left(0, z_{2}\right)} \\
& =\frac{1}{\lambda} \frac{\frac{\partial^{2}}{\partial z_{1} \partial z_{2}} H\left(\lambda_{1} F_{1}\left(z_{1}\right), \lambda_{2} F_{2}\left(z_{2}\right)\right)}{\frac{\partial}{\partial z_{2}} \bar{F}_{2}\left(z_{2}\right)} .
\end{aligned}
$$

Substituting $\bar{F}_{i}\left(x_{i}\right)=U_{i}\left(x_{i}\right) / \lambda_{i}$, we get

$$
\begin{aligned}
\cdots & =\frac{\lambda_{2}}{\lambda} \frac{\left.h\left(u_{1}, u_{2}\right)\right|_{\substack{u_{1}=U_{1}\left(z_{1}\right) \\
u_{2}=U_{2}\left(z_{2}\right)}} \frac{\partial}{\partial z_{1}} U_{1}\left(z_{1}\right) \frac{\partial}{\partial z_{2}} U_{2}\left(z_{2}\right)}{\frac{\partial}{\partial z_{2}} U_{2}\left(u_{2}\right)} \\
& =\left.\frac{\lambda_{2}}{\lambda} h\left(u_{1}, u_{2}\right)\right|_{\substack{u_{1}=U_{1}\left(z_{1}\right) \\
u_{2}=U_{2}\left(z_{2}\right)}} v_{1}\left(d z_{1}\right) .
\end{aligned}
$$




\section{B.2 Copula and $\sigma$-finite Lévy measures}

Let us assume that the $v_{1}$ and $v_{2}$ are two Lévy measures with infinite measure.

Let $v$ be a $\sigma$-finite Lévy measure and $L$ the corresponding Lévy process. Here we consider $L$ with only positive jumps. Cutting off the jumps smaller than $\varepsilon$, the corresponding Lévy process $L^{\varepsilon}$ can be written as follows.

$$
L^{\varepsilon}:=\sum_{i=1}^{N_{\varepsilon}(t)} Y_{i, \varepsilon},
$$

where $N_{\varepsilon}$ is a Poisson point process with parameter $v\left(\mathbb{R}_{+}^{2} \backslash(0, \varepsilon) \times(0, \varepsilon)\right)$ and $\left\{Y_{i, \varepsilon}: i \in \mathbb{N}\right\}$ are independent identical distributed random variables with survival function

$$
\bar{F}_{\varepsilon}(x, y)=\frac{U(x, y)}{U(\varepsilon, \varepsilon)}, \quad x, y \geq \varepsilon .
$$

Now, the aim is to express the survival copula of the two dimensional random variable $Y_{i, \varepsilon}$ by the Lévy copula $H$ and vice versa. The survival copula $\bar{C}_{\varepsilon}$ of $Y_{i, \varepsilon}$ is given by

$$
\bar{C}_{\varepsilon}(u, v)=\bar{F}_{\varepsilon}\left(\bar{F}_{1, \varepsilon}^{-1}(u), \bar{F}_{2, \varepsilon}^{-1}(u)\right), \quad u, v \in[0,1] .
$$

Since

$$
\bar{F}_{i, \varepsilon}(x)=\frac{U_{i, \varepsilon}(x)}{U_{i}(\varepsilon)}, \quad i=1,2,
$$

where $U_{i, \varepsilon}(x)=v_{i}([x, \infty))$ for $x \geq \varepsilon$. It follows that

$$
\bar{F}_{i, \varepsilon}^{-1}(u)=U_{i, \varepsilon}^{-1}\left(U_{i}(\varepsilon) u\right), \quad u \in[0,1], i=1,2 .
$$

Therefore,

$$
\bar{C}_{\varepsilon}(u, v)=\bar{F}_{\varepsilon}\left(U_{1, \varepsilon}^{-1}\left(U_{1}(\varepsilon) u\right), U_{2, \varepsilon}^{-1}\left(U_{2}(\varepsilon) v\right)\right) .
$$

Next, (B.5) implies that

$$
\bar{C}_{\varepsilon}(u, v)=\frac{U\left(U_{1, \varepsilon}^{-1}\left(U_{1}(\varepsilon) u\right), U_{2, \varepsilon}^{-1}\left(U_{2}(\varepsilon) v\right)\right)}{U(\varepsilon, \varepsilon)} .
$$

Finally, by the definition of $H$ we get

$$
\bar{C}_{\varepsilon}(u, v)=\frac{H\left(U_{1}(\varepsilon) u, U_{2}(\varepsilon) v\right)}{H\left(U_{1}(\varepsilon), U_{2}(\varepsilon)\right)} .
$$

In case $v_{1}=v_{2}$, we get by the scaling property of the Clayton copula (see Example B.1)

$$
\bar{C}_{\varepsilon}(u, v)=\frac{U_{1}(\varepsilon)}{U_{1}(\varepsilon) H(1,1)} H(u, v)=H(u, v) .
$$

This means that the survival copula $\bar{C}_{\varepsilon}$ is given by $H$. 
Proposition B.2. Let us assume the copula satisfies the following scaling property

$$
H\left(\alpha u_{1}, \alpha u_{2}\right)=\alpha H\left(u_{1}, u_{2}\right), \quad u_{1}, u_{2} \in \mathbb{R} .
$$

Let us define

$$
h\left(u_{1}, u_{2}\right):=\frac{\partial^{2}}{\partial u_{1} \partial u_{2}} H\left(u_{1}, u_{2}\right) .
$$

Then the conditional probability of $Y_{1, \varepsilon}$ given $Y_{2, \varepsilon}$ is

$$
\mathbb{P}\left(Y_{1, \varepsilon}=z_{1} \mid Y_{2, \varepsilon}=z_{2}\right)=\left.h\left(u_{1}, u_{2}\right)\right|_{\substack{u_{1}=U_{1}\left(z_{1}\right) \\ u_{2}=U_{2}\left(z_{2}\right)}} v\left(d z_{1}\right),
$$

for the case where $v_{1}=v_{2}$.

Proof. The formula can be shown by direct calculations. In particular, we can argue along the following lines

$$
\mathbb{P}\left(Y_{1, \varepsilon}=z_{1} \mid Y_{2, \varepsilon}=z_{2}\right)=\frac{\mathbb{P}\left(\left\{Y_{1, \varepsilon}=z_{1}\right\} \cap\left\{Y_{2, \varepsilon}=z_{2}\right\}\right)}{\mathbb{P}\left(\left\{Y_{2, \varepsilon}=z_{2}\right\}\right)}=\frac{\frac{\partial^{2}}{\partial z_{1} \partial z_{2}} \bar{F}_{\varepsilon}\left(z_{1}, z_{2}\right)}{\frac{\partial}{\partial z_{2}} \bar{F}_{\varepsilon}\left(0, z_{2}\right)} .
$$

Owing to the following equalities

$$
\frac{\partial}{\partial z_{2}} \bar{F}_{\varepsilon}\left(0, z_{2}\right)=\frac{\partial}{\partial z_{2}} \bar{F}_{2, \varepsilon}\left(z_{2}\right)=\frac{\frac{\partial}{\partial z_{2}} U_{2}\left(z_{2}\right)}{U_{2}(\varepsilon)}=\frac{\nu_{2}\left(z_{2}\right)}{U_{2}(\varepsilon)},
$$

and the scaling property (B.6) we get

$$
\begin{aligned}
\mathbb{P}\left(Y_{1, \varepsilon}\right. & \left.=z_{1} \mid Y_{\varepsilon, 2}=z_{2}\right) \\
& =\left.\frac{\partial^{2}}{\partial u_{1} \partial u_{2}} H\left(u_{1}, u_{2}\right)\right|_{\substack{u_{1}=\bar{F}_{\varepsilon, 1}\left(z_{1}\right) \\
u_{2}=\bar{F}_{\varepsilon, 2}\left(z_{2}\right)}} \frac{\frac{\partial}{\partial z_{1}} \bar{F}_{1, \varepsilon}\left(z_{1}\right) \frac{\partial}{\partial z_{2}} \bar{F}_{2, \varepsilon}\left(z_{2}\right)}{\frac{\partial}{\partial z_{2}} \bar{F}_{2, \varepsilon}\left(z_{2}\right)} \\
& =\left.2^{1 / \theta} \frac{\partial^{2}}{\partial u_{1} \partial u_{2}} H\left(u_{1}, u_{2}\right)\right|_{\substack{u_{1}=U_{1}\left(z_{1}\right) / U_{1}(\varepsilon) \\
u_{2}=U_{2}\left(z_{2}\right) / U_{2}(\varepsilon)}} \frac{\partial}{\partial z_{1}} \bar{F}_{1, \varepsilon}\left(z_{1}\right) \\
& =\left.2^{1 / \theta} h\left(u_{1}, u_{2}\right)\right|_{\substack{u_{1}=U_{1}\left(z_{1}\right) \\
u_{2}=U_{2}\left(z_{2}\right)}} v_{1}\left(d z_{1}\right) .
\end{aligned}
$$

Example B.2. As mentioned in Example B.1, the Clayton copula is given by

$$
H\left(u_{1}, u_{2}\right)=\left(\frac{1}{2} u_{1}^{-\theta}+\frac{1}{2} u_{2}^{-\theta}\right)^{-\frac{1}{\theta}} \beta 1_{u_{1} u_{2}>0}, \quad u_{1}, u_{2} \geq 0 .
$$

A short calculation shows that for $i=1,2$

$$
\frac{\partial H\left(u_{1}, u_{2}\right)}{\partial u_{i}}=\frac{1}{2}\left(\frac{1}{2} u_{1}^{-\theta}+\frac{1}{2} u_{2}^{-\theta}\right)^{-\frac{1}{\theta}-1} u_{i}^{-\theta-1}
$$


and

$$
\frac{\partial^{2} H\left(u_{1}, u_{2}\right)}{\partial u_{1} \partial u_{2}}=\frac{1}{4}(1+\theta)\left(\frac{1}{2} u_{1}^{-\theta}+\frac{1}{2} u_{2}^{-\theta}\right)^{-\frac{1}{\theta}-2} u_{1}^{-\theta-1} u_{2}^{-\theta-1} .
$$

Therefore

$$
h\left(u_{1}, u_{2}\right)=\frac{1}{4}(1+\theta)\left(\frac{1}{2} u_{1}^{-\theta}+\frac{1}{2} u_{2}^{-\theta}\right)^{-\frac{1}{\theta}-2} u_{1}^{-\theta-1} u_{2}^{-\theta-1},
$$

which implies that

$$
\begin{aligned}
& \mathbb{P}\left(\Delta L_{1}(t)=z_{1} \mid \Delta L_{2}(t)=z_{2}\right) \\
& \quad=\left.\frac{1}{4}(1+\theta)\left(\frac{1}{2} u_{1}^{-\theta}+\frac{1}{2} u_{2}^{-\theta}\right)^{-\frac{1}{\theta}-2} u_{1}^{-\theta-1} u_{2}^{-\theta-1}\right|_{\substack{u_{1}=U_{1}\left(z_{1}\right) \\
u_{2}=U_{2}\left(z_{2}\right)}} v_{1}\left(d z_{1}\right) .
\end{aligned}
$$

\section{Appendix C: Application of Lévy-upward theorem}

Before we start our main theorem of this section, we will illustrate the following remark which is useful to complete the proof of the main theorem of this section.

Remark C.1. Let $(\Omega, \mathcal{A}, \mu)$ be a measure space and $\mathcal{L} \subset \mathcal{A}$. We say that $\mathcal{L}$ is a lattice, if $\mathcal{L}$ is closed under countable unions and intersections, and $\varnothing, \Omega \in \mathcal{L}$. Let $\mathcal{L}^{c}:=\{A \in \mathcal{A}, \Omega \backslash A \in \mathcal{L}\}$. By the definition of the $\sigma$-algebra, we know that if $\mathcal{L}$ is a $\sigma$-algebra, then $\mathcal{L}$ is also a lattice and $\mathcal{L}^{c}=\mathcal{L}$. Therefore, in case $\mathcal{L}$ is a $\sigma$-algebra, Theorem 3.1 in Robertson (1965) reads:

$$
\mathbb{E}_{\gamma}\left[\frac{1}{X} \mid \mathcal{L}\right]=\left(\mathbb{E}_{\mu}[X \mid \mathcal{L}]\right)^{-1},
$$

with $\gamma(A)=\int_{A} X(\omega) \mu(d \omega)$ and $X$ is a square integrable random variable.

Using Theorem 3.1 of Robertson (1965) and the Lévy's upward theorem we can show the following theorem.

Theorem C.1. Let $V=\{V(t): t \geq 0\}$ be a solution to equation (2.8) and $V^{\varepsilon}=$ $\left\{V^{\varepsilon}(t): t \geq 0\right\}, \varepsilon \in(0,1]$, be the family of a solutions to (2.22). Let $\left\{\Gamma_{\varepsilon}: \varepsilon \in\right.$ $(0,1)$ \} be a family of uniformly integrable stochastic processes. Fix $p=1$ or 2 . In particular, for any $t \geq 0$ the family $\left\{\left|\Gamma_{\varepsilon}(t)\right|^{4 p}: \varepsilon \in(0,1]\right\}$ is uniformly integrable and $\lim _{\varepsilon \rightarrow 0} \Gamma_{\varepsilon}(t)=\Gamma(t), \mathbb{Q}$-a.s. Then, we have $\mathbb{Q}$-a.s. and in $L^{1}(\Omega ; \mathbb{R})$

$$
\lim _{\varepsilon \rightarrow 0} \mathbb{E}^{\mathbb{Q}}\left|\mathbb{E}^{\mathbb{Q}_{\varepsilon}}\left[\Gamma_{\varepsilon}(t) V^{\varepsilon}(t) \mid \mathcal{Y}_{t}^{\varepsilon}\right]\right|^{p}=\left.\mathbb{E}^{\mathbb{Q}}\left[\Gamma(t) V(t) \mid \mathcal{Y}_{t}\right]\right|^{p},
$$

where

$$
\left.\frac{d \mathbb{Q}}{d \mathbb{Q}_{\varepsilon}}\right|_{\mathcal{F}_{t}}=\frac{V^{\varepsilon}(t)}{V(t)}, \quad t \geq 0
$$


Proof. Apply the Kallianpur-Striebel formula (Kallianpur and Striebel (1968)) to get

$$
\begin{aligned}
\mathbb{E}^{\mathbb{Q}}\left|\mathbb{E}^{\mathbb{Q}_{\varepsilon}}\left[\Gamma_{\varepsilon}(t) V^{\varepsilon}(t) \mid \mathcal{Y}_{t}^{\varepsilon}\right]-\mathbb{E}^{\mathbb{Q}}\left[\Gamma(t) V(t) \mid \mathcal{Y}_{t}\right]\right|^{p} \\
=\mathbb{E}^{\mathbb{Q}}\left|\frac{\mathbb{E}^{\mathbb{Q}}\left[\Gamma_{\varepsilon}(t) V(t) \mid \mathcal{Y}_{t}^{\varepsilon}\right]}{\mathbb{E}^{\mathbb{Q}}\left[\frac{V(t)}{V^{\varepsilon}(t)} \mid \mathcal{Y}_{t}^{\varepsilon}\right]}-\mathbb{E}^{\mathbb{Q}}\left[\Gamma(t) V(t) \mid \mathcal{Y}_{t}\right]\right|^{p} \\
\leq 2^{p-1} \mathbb{E}^{\mathbb{Q}}\left|\frac{\mathbb{E}^{\mathbb{Q}}\left[\Gamma_{\varepsilon}(t) V(t) \mid \mathcal{Y}_{t}^{\varepsilon}\right]}{\mathbb{E}^{\mathbb{Q}}\left[\frac{V(t)}{V^{\varepsilon}(t)} \mid \mathcal{Y}_{t}^{\varepsilon}\right]}-\frac{\mathbb{E}^{\mathbb{Q}}\left[\Gamma(t) V(t) \mid \mathcal{Y}_{t}\right]}{\mathbb{E}^{\mathbb{Q}}\left[\frac{V(t)}{V^{\varepsilon}(t)} \mid \mathcal{Y}_{t}^{\varepsilon}\right]}\right|^{p} \\
+2^{p-1} \mathbb{E}^{\mathbb{Q}}\left|\frac{\mathbb{E}^{\mathbb{Q}}\left[\Gamma(t) V(t) \mid \mathcal{Y}_{t}\right]}{\mathbb{E}^{\mathbb{Q}}\left[\frac{V(t)}{V^{\varepsilon}(t)} \mid \mathcal{Y}_{t}^{\varepsilon}\right]}-\mathbb{E}^{\mathbb{Q}}\left[\Gamma(t) V(t) \mid \mathcal{Y}_{t}\right]\right|^{p} .
\end{aligned}
$$

The Hölder inequality gives

$$
\begin{aligned}
\cdots \leq & 2^{p-1}\left(\mathbb{E}^{\mathbb{Q}}\left|\frac{1}{\mathbb{E}^{\mathbb{Q}}\left[\frac{V(t)}{V^{\varepsilon}(t)} \mid \mathcal{Y}_{t}^{\varepsilon}\right]}\right|^{2 p}\right)^{\frac{1}{2}} \\
& \times\left(\mathbb{E}^{\mathbb{Q}}\left|\mathbb{E}^{\mathbb{Q}}\left[\Gamma_{\varepsilon}(t) V(t) \mid \mathcal{Y}_{t}^{\varepsilon}\right]-\mathbb{E}^{\mathbb{Q}}\left[\Gamma(t) V(t) \mid \mathcal{Y}_{t}\right]\right|^{2 p}\right)^{\frac{1}{2}} \\
& +2^{p-1}\left(\mathbb{E}^{\mathbb{Q}}\left|\frac{\mathbb{E}^{\mathbb{Q}}\left[\Gamma(t) V(t) \mid \mathcal{Y}_{t}\right]}{\mathbb{E}^{\mathbb{Q}}\left[\frac{V(t)}{V^{\varepsilon}(t)} \mid \mathcal{Y}_{t}^{\varepsilon}\right]}\right|^{2 p}\right)^{\frac{1}{2}}\left(\mathbb{E}^{\mathbb{Q}}\left|\mathbb{E}^{\mathbb{Q}}\left[\frac{V(t)}{V^{\varepsilon}(t)} \mid \mathcal{Y}_{t}^{\varepsilon}\right]-1\right|^{2 p}\right)^{\frac{1}{2}} .
\end{aligned}
$$

Now we will show that for $\varepsilon \rightarrow 0$, the first term in last inequality converges to zero. First, we will show that there exists a constant $C>0$ such that

$$
\mathbb{E}^{\mathbb{Q}}\left|\frac{1}{\mathbb{E}^{\mathbb{Q}}\left[\frac{V(t)}{V^{\varepsilon}(t)} \mid \mathcal{Y}_{t}^{\varepsilon}\right]}\right|^{2 p}<C, \quad \varepsilon \in(0,1]
$$

By Theorem 3.1 in Robertson (1965), Jensen's inequality and Hölder inequality we get

$$
\begin{aligned}
\mathbb{E}^{\mathbb{Q}}\left|\frac{1}{\mathbb{E}^{\mathbb{Q}}\left[\frac{V(t)}{V^{\varepsilon}(t)} \mid \mathcal{Y}_{t}^{\varepsilon}\right]}\right|^{2 p} & =\mathbb{E}^{\mathbb{Q}}\left|\mathbb{E}^{\mathbb{Q}_{\varepsilon}}\left[\frac{V^{\varepsilon}(t)}{V(t)} \mid \mathcal{Y}_{t}^{\varepsilon}\right]\right|^{2 p} \\
& \leq \mathbb{E}^{\mathbb{Q}}\left(\mathbb{E}^{\mathbb{Q}_{\varepsilon}}\left[\left|\frac{V^{\varepsilon}(t)}{V(t)}\right|^{2 p} \mid \mathcal{Y}_{t}^{\varepsilon}\right]\right) \\
& \leq \mathbb{E}^{\mathbb{Q}_{\varepsilon}}\left(\frac{V^{\varepsilon}(t)}{V(t)} \mathbb{E}^{\mathbb{Q}_{\varepsilon}}\left[\left|\frac{V^{\varepsilon}(t)}{V(t)}\right|^{2 p} \mid \mathcal{Y}_{t}^{\varepsilon}\right]\right) \\
& \leq\left(\mathbb{E}^{\mathbb{Q}_{\varepsilon}}\left|\frac{V^{\varepsilon}(t)}{V(t)}\right|^{2}\right)^{\frac{1}{2}}\left(\mathbb{E}^{\mathbb{Q}_{\varepsilon}}\left|\frac{V^{\varepsilon}(t)}{V(t)}\right|^{4 p}\right)^{\frac{1}{2}} \\
& =\left(\mathbb{E}^{\mathbb{Q}}\left|\frac{V^{\varepsilon}(t)}{V(t)}\right|\right)^{\frac{1}{2}}\left(\mathbb{E}^{\mathbb{Q}}\left|\frac{V^{\varepsilon}(t)}{V(t)}\right|^{4 p-1}\right)^{\frac{1}{2}} .
\end{aligned}
$$


To see that the last terms are bounded, first, note that $V^{-1}=Z$ where $Z$ solves (2.6). Due to the fact that $g$ is bounded, $Z$ has bounded moments of order $8 p-2$. In addition, for any $t \geq 0, V(t)$ and $V^{\varepsilon}(t)$ have also uniform bounds of order $8 p-2$. Hence, we conclude the RHS above is uniformly for all $\varepsilon>0$ bounded.

Next, we would like to show that

$$
\lim _{\varepsilon \rightarrow 0} \mathbb{E}^{\mathbb{Q}}\left|\mathbb{E}^{\mathbb{Q}}\left[\Gamma_{\varepsilon}(t) V(t) \mid \mathcal{Y}_{t}^{\varepsilon}\right]-\mathbb{E}^{\mathbb{Q}}\left[\Gamma(t) V(t) \mid \mathcal{Y}_{t}\right]\right|^{2 p}=0
$$

For the notational convenient, take $\tilde{\Gamma}_{t}^{\varepsilon}=\Gamma_{\varepsilon}(t) V(t)$ and $\tilde{\Gamma}_{t}^{0}=\Gamma(t) V(t)$. For fixed positive $R>0$ (the exact value of $R$ we will fix later) we get

$$
\begin{aligned}
\mathbb{E}^{\mathbb{Q}}\left|\mathbb{E}^{\mathbb{Q}}\left[\Gamma_{t}^{\varepsilon} \mid \mathcal{Y}_{t}^{\varepsilon}\right]-\mathbb{E}^{\mathbb{Q}}\left[\Gamma_{t}^{0} \mid \mathcal{Y}_{t}\right]\right|^{2 p} \\
\leq 2^{p-1} \mathbb{E}^{\mathbb{Q}}\left|\mathbb{E}^{\mathbb{Q}}\left[\Gamma_{t}^{\varepsilon} \mathbf{1}_{\left|\Gamma_{t}^{\varepsilon}\right| \leq R} \mid \mathcal{Y}_{t}^{\varepsilon}\right]-\mathbb{E}^{\mathbb{Q}}\left[\Gamma_{t}^{0} \mathbf{1}_{\left|\Gamma_{t}^{0}\right| \leq R} \mid \mathcal{Y}_{t}\right]\right|^{2 p} \\
\quad+2^{p-1} \mathbb{E}^{\mathbb{Q}}\left|\mathbb{E}^{\mathbb{Q}}\left[\Gamma_{t}^{\varepsilon} \mathbf{1}_{\left|\Gamma_{t}^{\varepsilon}\right|>R} \mid \mathcal{Y}_{t}^{\varepsilon}\right]-\mathbb{E}^{\mathbb{Q}}\left[\Gamma_{t}^{0} \mathbf{1}_{\left|\Gamma_{t}^{0}\right|>R} \mid \mathcal{Y}_{t}\right]\right|^{2 p} \\
\leq 2^{p-1} \mathbb{E}^{\mathbb{Q}}\left|\mathbb{E}^{\mathbb{Q}}\left[\Gamma_{t}^{\varepsilon} \mathbf{1}_{\left|\Gamma_{t}^{\varepsilon}\right| \leq R} \mid \mathcal{Y}_{t}^{\varepsilon}\right]-\mathbb{E}^{\mathbb{Q}}\left[\Gamma_{t}^{0} \mathbf{1}_{\left|\Gamma_{t}^{0}\right| \leq R} \mid \mathcal{Y}_{t}\right]\right|^{2 p} \\
\quad+2^{2 p-2} \mathbb{E}^{\mathbb{Q}}\left|\Gamma_{t}^{\varepsilon} \mathbf{1}_{\left|\Gamma_{t}^{\varepsilon}\right|>R}\right|^{2 p}+2^{2 p-2} \mathbb{E}^{\mathbb{Q}}\left|\Gamma_{t}^{0} \mathbf{1}_{\left|\Gamma_{t}^{0}\right|>R}\right|^{2 p}
\end{aligned}
$$

The last inequality holds due to the Jensen's inequality. Since for any $t \geq 0$, the family $\left\{\left|\Gamma_{t}^{\varepsilon}\right|^{2 p}: \varepsilon \in(0,1]\right\}$ is uniformly integrable, for any $\kappa>0$ there exist a number $R>0$ such that for all $\varepsilon \in(0,1]$,

$$
\mathbb{E}^{\mathbb{Q}}\left|\Gamma_{t}^{\varepsilon} \mathbf{1}_{\left|\Gamma_{t}^{\varepsilon}\right|>R}\right|^{2 p}<\frac{\kappa}{4}
$$

and

$$
\mathbb{E}^{\mathbb{Q}}\left|\Gamma_{t}^{0} \mathbf{1}_{\left|\Gamma_{t}^{0}\right|>R}\right|^{2 p}<\frac{\kappa}{4}
$$

Let $R>0$ be fixed. First,

$$
\begin{aligned}
\mathbb{E}^{\mathbb{Q}}\left|\mathbb{E}^{\mathbb{Q}}\left[\Gamma_{t}^{\varepsilon} \mathbf{1}_{\left|\Gamma_{t}^{\varepsilon}\right| \leq R} \mid \mathcal{Y}_{t}^{\varepsilon}\right]-\mathbb{E}^{\mathbb{Q}}\left[\Gamma_{t}^{0} \mathbf{1}_{\left|\Gamma_{t}^{0}\right| \leq R} \mid \mathcal{Y}_{t}\right]\right|^{2 p} \\
\quad \leq R^{2 p-1} \mathbb{E}^{\mathbb{Q}}\left|\mathbb{E}^{\mathbb{Q}}\left[\Gamma_{t}^{\varepsilon} \mathbf{1}_{\left|\Gamma_{t}^{\varepsilon}\right| \leq R} \mid \mathcal{Y}_{t}^{\varepsilon}\right]-\mathbb{E}^{\mathbb{Q}}\left[\Gamma_{t}^{0} \mathbf{1}_{\left|\Gamma_{t}^{0}\right| \leq R} \mid \mathcal{Y}_{t}\right]\right|
\end{aligned}
$$

By the Lévy-Upward theorem (see page 196 in Dembo (2012)), there exist a number $\varepsilon_{1}>0$, such that for all $\varepsilon \in\left(0, \varepsilon_{1}\right]$,

$$
R^{2 p-1} \mathbb{E}^{\mathbb{Q}}\left|\mathbb{E}^{\mathbb{Q}}\left[\Gamma_{t}^{\varepsilon} \mathbf{1}_{\left|\Gamma_{t}^{\varepsilon}\right| \leq R} \mid \mathcal{Y}_{t}^{\varepsilon}\right]-\mathbb{E}^{\mathbb{Q}}\left[\Gamma_{t}^{0} \mathbf{1}_{\left|\Gamma_{t}^{0}\right| \leq R} \mid \mathcal{Y}_{t}\right]\right|<\frac{\kappa}{2}
$$

This implies that for all $\varepsilon \in\left(0, \varepsilon_{1}\right]$,

$$
\mathbb{E}^{\mathbb{Q}}\left|\mathbb{E}^{\mathbb{Q}}\left[\Gamma_{t}^{\varepsilon} \mid \mathcal{Y}_{t}^{\varepsilon}\right]-\mathbb{E}^{\mathbb{Q}}\left[\Gamma_{t}^{0} \mid \mathcal{Y}_{t}\right]\right|^{2 p}<\kappa
$$


This gives Claim (C.4). Combining results (C.3) and (C.4), implies that the first term in last inequality of (C.2) goes to zero as $\varepsilon \rightarrow 0$. It remains to show

$$
\left|\mathbb{E}^{\mathbb{Q}}\left[\frac{V(t)}{V^{\varepsilon}(t)} \mid \mathcal{Y}_{t}^{\varepsilon}\right]-1\right|^{2 p} \rightarrow 0 \quad \text { as } \varepsilon \rightarrow 0 .
$$

By similar arguments, we can prove that the term above also converges to zero as $\varepsilon \rightarrow 0$, which gives the assertion.

\section{Acknowledgments}

The authors are very thankful to the anonymous referee for his/her insightful comments and remarks, which improve the manuscript. We are also very grateful to Professor Dan Crisan for his valuable suggestions and comments for improving the manuscript. This work was supported by the Austrian Science foundation (FWF), Projectnumber P17273-N12.

\section{References}

Applebaum, D. (2009). Lévy Processes and Stochastic Calculus, 2nd ed. Cambridge Studies in Advanced Mathematics. Cambridge: Cambridge University Press. MR2512800

Bain, A. and Crisan, D. (2009). Fundamentals of Stochastic Filtering. Stochastic Modeling and Applied Probability 60. New York: Springer. MR2454694

Borkar, V. S. (1989). Optimal Control of Diffusion Processes. Harlow, United Kingdom: Longman Group UK Limited.

Brémaud, P. (1981). Point Processes and Queues: Martingale Dynamics, 1st ed. Springer Series in Statistics. New York: Springer. MR0636252

Ceci, C. (2006). Risk minimizing hedging for a partially observed high frequency data model. An International Journal of Probability and Stochastic Processes 78, 13-31. MR2219710

Ceci, C. and Colaneri, K. (2012). Nonlinear filtering for jump diffusion observations. Advances in Applied Probability 44, 678-701. MR3024605

Cherubini, U., Luciano, E. and Vecchiato, W. (2004). Copula Methods in Finance, 1st ed. Wiley Finance Series. Chichester: John Wiley \& Sons Ltd.

Cont, R. and Tankov, P. (2004). Financial Modeling with Jump Processes, 1st ed. Chapman \& Hall/CRC Financial Mathematics Series. Boca Raton, Florida: Chapman \& Hall/CRC. MR2042661

Cont, R. and Tankov, P. (2006). Retrieving Lévy processes from option prices: Regularization of an ill-posed inverse problem. SIAM Journal on Control and Optimization 45, 1-25. MR2225295

DaPrato, G. and Zabczyk, J. (1992). Stochastic Equations in Infinite Dimensions. Cambridge: Cambridge Publisher. MR1207136

Dembo, A. (2012). Probability Theory: STAT310/MATH230.

Engel, K. J. and Nagel, R. (2000). One-Parameter Semigroups for Linear Evolution Equations, 1st ed. Berlin: Springer. MR1721989

Ethier, S. and Kurtz, T. (1986). Markov Processes: Characterization and Convergence. New York: John Wiley \& Sons, Inc. MR0838085

Fernando, B. P. W. and Sritharan, S. S. (2013). Nonlinear filtering of stochastic Navier-Stokes equation with Itô-Lévy noise. Stochastic Analysis and Applications 31, 381-426. 
Fernando Pani, W., Hausenblas, E. and Razafimandimby, P. A. (2017). Analytic properties of Markov semigroup generated by SDEs driven by Lévy processes. Potential Analysis 46, 1-21. MR3595959

Frey, R. and Runggaldier, W. (2001). A nonlinear filtering approach to volatility estimation with a view towards high frequency data. International Journal of Theoretical and Applied Finance 4, 199-210.

Frey, R. and Runggaldier, W. (2010). Pricing credit derivatives under incomplete information: A nonlinear-filtering approach. Finance and Stochastics 14, 495-526.

Frey, R. and Schimdt, T. (2012). Pricing and hedging of credit derivatives via the innovation approach to nonlinear filtering. Finance and Stochastics 16, 105-133.

Frey, R., Schimdt, T. and Xu, L. (2013). On Galerkin approximations for the Zakai equation with diffusive and point process observations. SIAM Journal on Numerical Analysis 51, 2036-2062. MR3077901

Getoor, R. K. (1975). On the construction of kernels. In Seminaire de Probabilities IX. Lecture Notes in Mathematics 465. Berlin: Springer.

Hausenblas, E. (2005). Existence, uniqueness and regularity of parabolic SPDEs driven by Poisson random measure. Electronic Journal of Probability 10, 1496-1546. MR2191637

Hausenblas, E. and Seidler, J. (2008). Stochastic convolutions driven by martingales: Maximal inequalities and exponential integrability. Stochastic Analysis and Applications 26, 98-119.

Hoh, W. (1998). A symbolic calculus for pseudo-differential operators generating Feller semigroups. Osaka Journal of Mathematics 35, 798-820.

Jacob, N. (2000). Pseudo Differential Operators and Markov Processes-I: Fourier Analysis and Semigroups, 1st ed. London: Imperial College Press.

Jacob, N. (2002). Pseudo Differential Operators and Markov Processes-II: Generators and Their Potential Theory, 2nd ed. London: Imperial College Press.

Jacob, N. (2005). Pseudo Differential Operators and Markov Processes-III: Markov Processes and Applications, 1st ed. London: Imperial College Press.

Kallenberg, O. (2002). Foundations of Modern Probability, 2nd ed. Probability and Its Applications. New York: Springer.

Kallianpur, G. and Striebel, C. (1968). Estimation of stochastic systems: Arbitrary system process with additive white noise observation errors. Annals of Mathematical Statistics 39, 785-801.

Kallsen, J. and Tankov, P. (2006). Characterization of dependence of multidimensional Lévy processes using Lévy copulas. Journal of Multivariate Analysis 97, 1551-1572. MR2275419

Kliemann, W. H., Koch, G. and Marchetti, F. (1990). On the un-normalized solution of the filtering problem with counting process observations. IEEE Transaction on Information Theory $\mathbf{3 6}, 1415-$ 1425.

Lipster, R. S. and Shiryaev, A. N. (1989). Theory of Martingales. Dordrecht: Kluwer Academic Publishers Group. MR1022664

Lipster, R. S. and Shiryaev, A. N. (2001). Statistics of Random Processes-II: Applications, 2nd ed. Berlin: Springer.

Malevergne, Y. and Sornette, D. (2006). Extreme Financial Risks from Dependence to Risk Management. Berlin: Springer. MR2191454

Nelsen, R. (2006). An Introduction to Copulas, 2nd ed. Springer Series in Statistics. New York: Springer. MR2197664

Robertson, T. (1965). A note on the reciprocal of the conditional expectation of a positive random variable. The Annals of Mathematical Statistics 36, 1302-1305.

Runst, T. and Sickel, W. (1996). Sobolev Spaces of Fractional Order, Nemytskij Operators and Nonlinear Partial Differential Equations. Berlin: De Gruyter. MR1419319 
Shubin, M. A. (2001). Pseudodifferential Operators and Spectral Theory, 1st ed. Berlin, Heidelberg: Springer. MR1852334

Stein, E. M. (1993). Harmonic Analysis: Real-Variable Methods, Orthogonality and Oscillatory Integrals. Princeton Mathematical Series 43. Monographs in Harmonic Analysis, III. Princeton, New Jersey: Princeton University Press.

\author{
Lehrstuhl für Angewandte Mathematik \\ Montanuniversität Leoben \\ Franz Josef Straße 18 \\ 8700 Leoben \\ Austria \\ E-mail: bandhisattambige.fernando@unileoben.ac.at \\ erika.hausenblas@unileoben.ac.at
}

